Revue

de Sémantique

et Pragmatique
Revue de Sémantique et Pragmatique

$35-36 \mid 2015$

Interface sémantique/morphologie

\title{
Interprétabilité ou grammaticalité ? les listèmes comme interface entre sémantique et morphologie
}

Interpretability or Grammaticality? Listemes at the semantics/morphology interface

\section{François Nemo}

\section{OpenEdition}

\section{Journals}

Édition électronique

URL : http://journals.openedition.org/rsp/1582

DOI : $10.4000 /$ rsp. 1582

ISSN : $2610-4377$

\section{Éditeur}

Presses universitaires d'Orléans

\section{Édition imprimée}

Date de publication : 1 mars 2015

Pagination : 105-144

ISSN : 1285-4093

Référence électronique

François Nemo, «Interprétabilité ou grammaticalité ? les listèmes comme interface entre sémantique et morphologie », Revue de Sémantique et Pragmatique [En ligne], 35-36 | 2015, mis en ligne le 01 mars 2016, consulté le 09 mai 2020. URL : http://journals.openedition.org/rsp/1582 ; DOI : https://doi.org/ 10.4000/rsp.1582 


\section{INTERPRÉTABILITÉ OU GRAMMATICALITÉ ? LES LISTĖMES COMME INTERFACE ENTRE SÉMANTIQUE ET MORPHOLOGIE}

François Nemo

Université d'Orléans, LLL, UMR 7270

\section{INTRODUCTION}

Interroger l'interface entre sémantique et morphologie ${ }^{1}$ suppose d'interroger d'abord les objectifs et ambitions respectives des deux disciplines et les intersections ou non de leur champs de recherche. Sachant que cette intersection peut être aussi bien inexistante (s'il y a autonomie), que réduite ou totale (s'il y a inclusion). Et qu'en débattre imposera in fine d'interroger la notion d'interprétabilité.

\subsection{DÉFINITION DE LA SÉMANTIQUE LINGUISTIQUE}

S'agissant de clarifier les objets respectifs des deux disciplines, il est possible de commencer par définir la sémantique linguistique comme « l'étude de l'ensemble des contraintes linguistiques qui pèsent sur l'interprétation », la sémantique n'étant pas définissable par une conception a priori de la nature du contenu communiqué - qui consisterait à poser par exemple que la sémantique aurait à étudier « ce que les phrases disent du monde ou leurs conditions de vérité »- mais doit l'être par la seule nature, linguistique ou non, des moyens utilisés (Nemo \& Cadiot, 1997a).

1 Je tiens à remercier les deux relecteurs anonymes dont les remarques auront je l'espère permis de rendre plus explicites des sections importantes de ce texte, et ce même si répondre à ces remarques légitimes a largement contribué à lui faite atteindre une taille sans doute trop importante. 


\subsection{CAHIER DES CHARGES DE LA SÉMANTIQUE LINGUISTIQUE}

S'agissant de définir le cahier des charges de toute sémantique, il est de même possible de montrer que celui-ci peut in fine être réduit à deux objectifs complémentaires, à savoir de :

- rendre compte exhaustivement de la diversité des emplois des signes et unités sémantiques, et des contraintes qui expliquent cette diversité;

- rendre compte des mécanismes qui régissent l'intégration sémantique, comme mise en relation interprétative des signes linguistiques entre eux, et ce, sous quelque forme que ce soit.

À charge pour le sémanticien de déterminer par ailleurs la nature des relations éventuelles pouvant exister entre ces deux questions.

\subsection{DÉFINITION, OBJECTIFS ET CAHIER DES CHARGES DE LA MORPHOLOGIE}

La morphologie peut être définie comme "l'étude des mécanismes qui régissent la formation et la forme des mots (ou des unités lexicales) » et concerne donc aussi bien la morphologie dérivationnelle et compositionnelle que flexionnelle, la morphophonologie que la morphosyntaxe, les relations d'allomorphie et de supplétion que le figement et l'idiomaticité supra-lexicale. Et ce même si certains termes, en particulier la notion de dérivation, peuvent le cas échéant prêter beaucoup à discussion.

\subsection{NON-AUTONOMIE DE LA MORPHOLOGIE PAR RAPPORT À LA SÉMANTIQUE}

À partir de là, il s'avère que rien de ce qu'entend donc traditionnellement décrire la morphologie ne se situe en réalité hors du champ du questionnement sémantique que nous venons de rappeler et qui a maintenant un demi-siècle. Ceci dans la mesure où en particulier - qu'il s'agisse de définir la nature des unités sémantiques et morphologiques ou de comprendre la façon dont elles sont associées - aucune raison a priori ne peut conduire à rendre obligatoire de supposer que des mécanismes sémantiques spécifiques soient en jeu en ce qui concerne par exemple les formes d'intégration sémantique qui sont en jeu entre les différents éléments d'un mot complexe ou encore la sémantique des temps/des modes/de l'aspect. Sachant par ailleurs que les unités lexicales, infralexicales (affixes, bases liées) et supra-lexicales (e.g. passer à tabac, tabasser) s'avèrent indubitablement polysémiques et polymorphes ${ }^{2}$. Moyennant quoi il n'est donc pas du tout étonnant qu'après avoir éprouvée leur méthodologie et leurs outils théoriques sur d'autres terrains, les sémanticiens spécialistes de la polysémie aient commencé au milieu des années 90 à travailler sur des objets qui jusque-là n'avaient intéressé que les grammairiens. Au moment même où

\footnotetext{
2 Comme l'illustre la polymorphie de forme et morph.
} 
certains morphologues (e.g. Lieber, 2002) en venaient à reconnaître le caractère central de la question de la polysémie pour toute morphologie.

Une fois que peut être ainsi apportée une réponse négative à la question théorique et empirique de savoir si les mécanismes à l'œuvre dans les domaines travaillés traditionnellement par la morphologie devraient être différents de ceux qui sont mobilisés en sémantique générale, il reste ensuite à savoir :

- quels outils et méthodes (au sens large) de la sémantique sont utiles et efficaces en morphologie?

- quels outils et méthodes (au sens large) de la morphologie pourraient être utiles à la sémantique?

et surtout à ce que les morphologues clarifient le rapport qu'ils revendiquent à la sémantique et au sémantique. Car si la syntaxe, pour le pire comme pour le meilleur, a formulé explicitement une conception du rapport entre syntaxe et sens qui a pu être falsifiée empiriquement, la morphologie (longtemps préoccupée par son seul rapport avec la syntaxe) n'a jamais tenu de discours homogène sur la façon dont se pose pour elle avec la question du sens linguistique. Moyennant quoi, il est possible de montrer que les débats entre linguistes se revendiquant sémanticiens et linguistes se revendiquant morphologues concerne moins la question de l'interface sémantique/morphologie que les hypothèses sémantiques, valides ou non, et les hypothèses linguistiques, là aussi valides ou non, que les uns comme les autres peuvent avancer. Ce qui dans les deux cas, mais en particulier dans le premier (voir section 1.7), fait qu'on ne peut pas plus concevoir une morphologie sémantiquement autonome qu'une sémantique commençant au niveau de la phrase.

\subsection{MORPHOLOGIE, SYNTAXE ET GRAMMATICALITÉ}

S'agissant du rapport syntaxe/morphologie, il faut signaler que si de nombreux linguistes de Saussure au premier Chomsky ont longtemps défini la syntaxe et la morphologie comme deux branches de la grammaire à traiter ensemble, la question d'une autonomie de la morphologie à l'intérieur de la grammaire s'est ensuite trouvée posée, au point d'assister par moment à un démembrement de la morphologie entre questions traitables ou non traitables sur la base des seuls mécanismes syntaxiques postulés (e.g. la percolation de traits et mécanisme X-barre pour Lieber, 1979).

\subsection{SYNTAXE ET SÉMANTIQUE}

S'agissant ensuite du rapport entre syntaxe et sémantique, il faut noter qu'après des débats internes très virulents au début des années 70, la syntaxe générative a proposé jusqu'aujourd'hui une théorie explicite de la relation entre syntaxe et sémantique selon laquelle la sémantique devait être définie 
comme l'étude de la part du sens qui se construit dans le module syntaxique en parallèle aux mécanismes syntaxiques, autrement dit au niveau des phrases. Et selon laquelle cette sémantique minimale était réductible au calcul de la forme propositionnelle/logique, celle-ci servant d'input au reste du processus d'interprétation (gouverné par la pragmatique). Ce qui revenait en nommant sémantique cette sémantique minimale, à identifier la sémantique à sa seule intersection avec la syntaxe. Et ce qui passait par un refus explicite de traiter des questions sémantiques à un autre niveau que celui de la phrase (et notamment de rendre compte de la polysémie des mots), une définition strictement combinatoire de l'intégration sémantique (compositionalité), et une ignorance quasi-complète de la complexité interne des mots.

Il faut ensuite noter que cette heuristique est aujourd'hui dans une impasse liée au triple fait de supposer l'existence d'un parallélisme logico-grammatical, d'une compositionalité stricte et d'une modularité ${ }^{3}$ du composant syntaxique. Et ce alors que le calcul de la forme propositionnelle s'est avéré reposer très largement sur des mécanismes pragmatiques (induisant le remplacement de la notion de forme propositionnelle par celle de forme logique). Alors que la sémantique des phrases s'est avérée massivement non-compositionnelle (cf. Pelletier (2004[1994], 150) ${ }^{4}$. Et alors que la justification en termes de modularité du composant linguistique de la réduction du sens des phrases à la seule forme logique (Borg 2004), parce qu'elle impose que toute forme d'interprétation ${ }^{5}$ observable hors des phrases ne puisse plus être maintenue dans la forme logique, conduit à ce que la quasi-totalité de l'interprétation conventionnelle des séquences phrastiques devienne imprédictible à partir de la forme logique, le minimalisme devenant sans cesse plus minimal.

On peut néanmoins reconnaître qu'en défendant une conception explicite et testable de l'interface syntaxe/sémantique, cette heuristique a permis en

3 La conception modulariste du « composant linguistique » impose que celui-ci soit cognitivement autonome, autrement dit qu'il fonctionne comme une boite noire et n'utilise jamais aucune information obtenue hors du module linguistique. L'application de ce principe en morphologie obligerait en toute rigueur à ne pas pouvoir décrire le fait que rouge-gorge puisse désigner autre chose qu'une gorge-rouge (et à considérer le fait que cela soit le cas comme non-linguistique). De même, si la désignation d'un oiseau était finalement acceptée, le fait qu'un oiseau tout rouge ne puisse être appelé rouge-gorge, ne pourrait pas être prédit, cette réalité résultant de la lexicalisation d'une contrainte pragmatique (Nemo, Portuguès, Petit, 2011).

${ }^{4}$ Notamment lorsque'il reconnaît que « that, together with giving up not our love but rather our blind adulation of Father Montague, will help us cheerfully embrace non-compositionality for the obvious truth that it is ».

5 Ainsi l'interprétation causale de «Paul qui était fatigué a été se coucher » ne peutelle être associée à la forme logique de cette phrase, cette relation se retrouvant au niveau discursif «Paul était fatigué. Il a été se coucher». 
étant lourdement falsifiée de prouver que les mécanismes sémantiques sont autonomes des mécanismes combinatoires et que l'interprétation sémantique est irréductible à l'interprétation vériconditionnelle (et parfois contradictoire avec celle-ci).

\subsection{MORPHOLOGIE ET SÉMANTIQUE}

La morphologie n'a pas défendu une conception explicite de son interface avec la sémantique, à la fois parce qu'elle ne pouvait s'appuyer sur le projet d'une sémantique formalisée, celle-ci étant une sémantique des phrases, et parce que confrontée empiriquement à l'instabilité du rapport forme/sens (Nemo, 2002b), elle a été bien en peine de proposer une modélisation robuste de celui-ci.

En laissant de côté les nombreuses approches de la morphologie ${ }^{6}$ qui en autonomie ou par emprunt d'outils ont abordé les questions sémantiques de façon très similaire à ce qui peut se pratiquer pour d'autres niveaux sémantiques, force est de constater que beaucoup de morphologues parmi les plus éminents tiennent des raisonnements sémantiques qui, à l'inverse, ne peuvent que laisser pantois le sémanticien. Et ce à la fois sur le fond, les questions principales et prioritaires étant oubliées, que sur la forme - tant sont nombreux les holdups anaphoriques - ou encore en ce qui concerne les méthodes, au travers du recours prioritaire ou exclusif à l'intuition, à une sémantique soustractive et à une pratique consistant à ne pas appliquer aux lexèmes les critères utilisés pour éliminer les morphèmes.

Si l'on dresse une liste des formes axiomatisés de raisonnement sémantique reprises depuis 40 ans en morphologie et qui apparaissent sémantiquement inacceptables, il faut relever pour ce qui concerne les diagnostics sémantiques qu'impose le constat de l'instabilité sémantique des unités sémantiques? et notamment des morphèmes, le fait de i) ne pas envisager sérieusement l'homonymie ; ii) réduire la polysémie à la seule variation métonymique ; iii) croire qu'il serait scientifiquement acceptable de décider ce qui dans le sens est fondamental ou pas, et de trier parmi les différentes strates sémantiques observables; iv) confondre le fait que le sens soit instable et le fait qu'il n'y ait rien de stable dans le sens, les deux questions étant sans rapport ; v) recourir en priorité à l'intuition pour déterminer le sens d'une unité, et considérer ensuite que ce qu'elle n'explique pas n'a pas de sens ; vi) croire qu'il est possible d'obtenir le sens d'une unité par soustraction, et quand la démarche

${ }^{6}$ On peut penser notamment au travail mené par Danielle Corbin ou dans son sillon.

7 Question qui est l'une des deux missions fondamentales de toute sémantique linguistique et où d'immenses progrès ont été faits depuis les travaux fondateurs de Sapir ([1944] 1963) et Benveniste ([1953] 1969). 
échoue, en conclure qu'elle n'a pas de sens ; vii) ignorer la question cruciale de la polycatégorialité ; viii) poser constamment des équivalences supposées entre des notions clairement distinctes et souvent contradictoires; ix) ignorer des descriptions sémantiques efficaces en attribuant aux contre-exemples un statut théorique et empirique exorbitant.

Ce qui permet ensuite de s'attaquer à des questions réellement difficiles et importantes pour l'interface sémantique/morphologie et aux premières avancées en ce domaine, qui concernent :

- l'idée selon laquelle les morphèmes sont à la fois les briques combinatoires de la langue (car obtenus par décomposition) et ses briques sémantiques, qui s'avère ne pas résister à l'analyse ;

- le fait que les morphèmes sont des briques purement sémantiques qui ne sont associés à aucune information combinatoire ni à aucun sens, mais seulement à des significations ;

- le fait que les morphèmes codent des contraintes sémantiques à satisfaire alors que les lexèmes sont associés aux différentes façons de les satisfaire (et sont pour cette raison polysémiques);

- l'idée selon laquelle la présence d'un morphème dans un mot ne peut être reconnue que si l'on est capable de décomposer exhaustivement celui-ci, qui se trouve contredite par l'existence des segments "non significatifs » n'interdisant en rien l'interprétation des autres éléments ;

- la croyance selon laquelle la signification d'un morphème devrait décrire la construction dans laquelle il est inséré. Alors qu'il s'avère que la signification d'un morphème ne préfigure jamais son contexte d'insertion et qu'il faut donc séparer strictement la description des morphèmes de celles des constructions dans lesquelles ils sont insérés.

Pour commencer par notre première liste, il faut avant tout comprendre la façon dont en sémantique se pose la question de l'association de plusieurs sens avec une forme signifiante, à savoir exclusivement en termes de choix entre dégroupement homonymique et polysémie ${ }^{8}$. Et constater qu'au lieu

${ }^{8}$ Sachant par ailleurs que la question de savoir si une forme est signifiante est toute autre, puisque imposant, en l'absence supposée de sens de justifier de l'autonomie de la forme concernée (et donc du caractère non arbitraire du découpage lui-même) et imposant pour postuler une unité signifiante l'existence démontrable d'une contribution de cette unité (d'une façon ou d'une autre) au processus interprétatif. Démarche qui n'impose pas en réalité d'identifier le signifié mais seulement de reconnaître son existence. Ne pas découpler le problème de la signifiance, que le linguiste de terrain peut trancher, de la question de la signification, que seul le sémanticien peut clarifier au terme d'un travail méthodique et assez long, est sans aucun doute le défaut sémantique majeur des méthodes descriptives de la linguistique, puisqu'elle conduit toujours à promouvoir au statut de signifié ce qui n'est qu'une valeur d'emploi du signe concerné. 
de trancher les questions qui se posent à partir de ces deux alternatives, une partie de la morphologie a choisi depuis 40 ans de substituer à l'alternative « homonymie/polysémie », une alternative singulière entre "sens saussurien fixe » et « absence de sens », aggravée par le fait que le « sens saussurien fixe » est par ailleurs conçu comme dénotatif/intuitif.

S'agissant ensuite de prendre au sérieux la polysémie - autrement dit le fait que l'existence de la variation sémantique n'implique pas l'absence de signification constante, par exemple quand une contrainte sémantique unique (la signification) se trouve pouvoir être satisfaite de différentes façons (les sens) - force est de constater que tel n'est pas le cas ${ }^{9}$ sauf pour la réduire à la seule métonymie désignationnelle (e.g. " j'ai pris un verre ») ${ }^{10}$, et ce cinquante ans après la distinction benvenistienne entre désignation et signification et plusieurs décennies de traitement efficace de la polysémie dans le cadre post-benvenistien des sémantiques instructionnelles et indicationnelles.

S'agissant du fait de croire qu'il serait possible scientifiquement de décider ce qui dans le sens est fondamental ou pas, il faut noter que vouloir hiérarchiser les strates sémantiques en privilégiant par exemple la valeur dénominative (e.g. le fait que rouge-gorge soit le nom d'un oiseau) est en morphologie particulièrement absurde puisque cela conduirait à nier le fait que rougegorge veut d'abord et avant tout dire gorge-rouge et à privilégier le fait que ce syntagme a été accidentellement employé à propos d'une espèce d'oiseau dont il est devenu le nom. La vérité étant que dans une science exacte, tout emploi de notions comme sens « fondamental » ou «normal » ne peut avoir la moindre justification scientifique ${ }^{11}$, surtout pour transformer (Aronoff \& Fudeman, $2005,129)$ une théorie extensionnelle de la signification ${ }^{12}$ massivement falsifiée en axiome analytique.

9 Aronoff $(1976,14)$ pratique pour s'opposer à la thèse de l'allo-meaning un procédé rhétorique nommé exagération absurdifiante (e.g. accuser quelqu'un de vouloir supprimer l'école quand il dit que les programmes sont surchargés) qui témoigne toujours d'une incapacité à invalider une thèse sous sa forme réelle.

10 A l'instar des alternances "mass/count", "figure-ground reversal”, "containercontained alternation", "people alternation, "characteristic-person alternation" citées par Aronoff \& Fudeman (2005, 129).

${ }^{11}$ Ne serait-ce que parce que toute affirmation de ce genre est infalsifiable.

12 Dire que l'aspect le plus fondamental du sens d'un mot est de référer et que reptile réfère « à tous les individus qui sont des reptiles », c'est non seulement confondre (Cadiot \& Nemo, 1997a, 1997b) la question « que veut dire X ? » et la question « qu'est-ce qu'un $\mathrm{X}$ ? », mais aussi confondre nouns et names en oubliant que les premiers ne sont aucunement réductible aux seconds. La signification de rept, dans reptile comme dans reptation, décrit un type de mouvement (i.e. ramper) et ne permet en rien à elle seule de déterminer ce qui sera un reptile ou non : la langue ne dit pas le monde, elle se contente de l'étiqueter, ce qui implique de décrire l'étiquette et d'oublier très largement ce qui est étiqueté. 
De même, le fait de recourir à l'intuition pour déterminer le sens d'une unité et en conclure ensuite que ce qu'elle n'explique pas n'a pas de sens est-il scientifiquement inacceptable. Car pas plus que la physique n'est une théorie de ce que les humains pensent du monde, la sémantique ne peut être une théorie de l'intuition sémantique des sujets parlants. Poser l'intuition comme boussole scientifique, dire ou croire par exemple que le sens de re- en français serait de nouveau parce que c'est ce que dit spontanément un locuteur du français, c'est croire que seule la conscience sémantique compte. Or tel n'est pas le cas, comme en témoigne l'embarras d'un locuteur francophone pour expliquer ce que veut dire télé- en français dans télévision, téléphone, télépathie, et comme l'a montré surtout la sémantique linguistique à chaque fois que la signification d'un signe a pu être identifiée : la signification n'est jamais intuitive et souvent contre-intuitive comme nous le verrons dans un instant avec re-.

Sont aussi inacceptables les approches soustractives (Nemo, 2002b) qui consistent naïvement à croire que le sens d'une unité U2 associée à une unité U1 pour former une unité U3 - que U2 soit un affixe ou une base - pourra être obtenu en retranchant du sens de U3 le sens de U1. Et ce d'abord dans la mesure où une telle démarche conduit invariablement conduit à obtenir pour U2 un sens qui va varier d'un U3 à un autre, et ensuite parce qu'elle masque tout aussi invariablement la contrainte sémantique constante qui sera associée à U2. En décrivant ainsi le sens de -ier dans pétrolier comme « bateau transportant du $\mathrm{U} 1$ » et prisonnier comme « personne enfermée dans une $\mathrm{U} 1$ », et en négligeant le fait qu'une relation « $\mathrm{X}$ est dans $\mathrm{Y}$ » constante ${ }^{13}$ existe sous la forme « U1 est dans $\mathrm{U} 3$ » pour pétrolier et « $\mathrm{U} 3$ est dans $\mathrm{U} 1$ » pour prisonnier. En croyant pour le morphème re-que parce que relire veut parfois dire "lire une nouvelle fois quelque chose » alors re- devrait vouloir dire «de nouveau» ou "une nouvelle fois ». Alors que nous aurons l'occasion de montrer que cette démarche soustractive masque la signification et force toujours le morphologue à choisir parmi les résultats de toutes ses soustractions un sens plus fondamental que les autres.

Un peu plus compréhensible tout en restant fautif est ensuite le fait de confondre « instabilité du sens d'une unité » et «absence de quelque chose de stable dans le sens des différents emplois de cette unité », alors qu'une des avancées principales de la sémantique depuis quarante ans a été non seulement de comprendre que de la variation pouvait coexister avec de l'invariance, mais

13 En l'espèce, ce scénario n'est pas la signification de -ier, mais un profilage de celleci (Cadiot \& Visetti, 2001), autrement dit une variante de la signification qui est partagée par des centaines d'emplois. La description par la glose back de la signification de re-par E. Williams (1973), citée dans Aronoff (1976) est elle aussi un profil partagé par des centaines d'emplois de re- en anglais. 
en fait qu'elle en était le résultat, par sous-détermination sémiologique du sens ${ }^{14}$.

S'agissant enfin de la question de la polycatégorialité, il n'est pas non plus acceptable d'axiomatiser une définition du morphème qui le dote automatiquement de propriétés grammaticales ${ }^{15}$. Et ce dès lors qu'il y a convergence depuis au moins vingt ans entre les résultats de la typologie des classes de mots (e.g. Hengeveld, 1992) et ceux de la sémantique linguistique sur le caractère non-catégoriel des unités porteuses de signification. Avec dans le premier cas, la reconnaissance du fait que dans beaucoup de langues à lexique non-catégoriel, les unités sémantiques de base n'ont pas de propriété grammaticale inhérente, autrement dit ne deviennent des termes, des prédicats, des modifieurs de prédicats ou des modifieurs de terme que quand elles sont employées, et du fait de cette insertion grammaticale. Et dans le second, qui concerne cette fois des langues à lexique dite catégoriel, du fait que les recherches sémantiques sur les unités polycatégorielles ont répondu à la question de savoir si la signification était catégorielle ou non par la négative, confortant ainsi la réponse esquissée dès la première formulation explicite de cette question chez Benveniste ([1953]1969). Pour ne donner en effet qu'un exemple de la façon dont un ensemble de lexèmes relevant de catégories syntaxiques diverses peuvent être expliqués comme étant tous les formes lexicalisées des emplois d'un unique morphème, il est possible de considérer la large polycatégorialité de but (Nemo, 2006b) et even (Nemo, 2007) en anglais, qui les conduit à prendre des sens aussi catégoriellement variés que mais, sauf, sans, presque, seulement pour le premier, et que même, plat (pour une surface), calme (pour une voix), régulier, uniforme, à égalité (pour un score), pair (pour un nombre), être quitte, exactement, égaliser, s'équilibrer, aplanir, encore, etc. pour le second. Et de prendre acte de la démonstration, pour chacune des deux séries, du fait que ces sens ne sont en réalité que des façons distinctes de satisfaire une unique contrainte morphémique.

14 Au point que les sens dits opposés tendent aujourd'hui à être conçus comme des moyen privilégiés d'accès à la signification. A l'instar des emplois opposés de enfin dits de soulagement (le enfin «ouf») et d'irritation (taisez-vous enfin), qui s'avèrent n'être en réalité que deux variantes d'un même scénario, avec dans un premier temps un problème qui se pose et dans un second temps une résolution du problème en question, la seule différence étant que le premier reçoit du point de vue pragmatique une lecture doublement constative (un problème s'est posé en $t_{-2}$, il est résolu en $t_{-1}$ ), alors que le second reçoit lui une lecture constative (un problème se pose en $\mathrm{t}_{0}$ ) et directive (un terme doit être mis au problème en $\mathrm{t}_{+1}$ ). Ce scénario se retrouvant sous différentes formes dans tous les emplois de enfin est la contrainte sémantique qui doit être satisfaite dans tous ses emplois.

15 Aronoff $(2005,4)$ défend ainsi l'idée qu'il est "important to take seriously the idea that the grammatical function of a morpheme, which may include its meaning, must be constant" là où la sémantique de la polycatégorialité défend l'idée que les able et to be able to et de transportable sont a priori le même morphème dans deux positions distinctes, à charge ensuite de prouver qu'il code bien une contrainte sémantique constante. 
Plus important encore pour l'analyse morphologique est la coexistence de lexèmes et de morphèmes dans la représentation sémantico-morphologique de ce que l'on avait coutume d'appeler des morphèmes libres, par exemple pour le morphème leven/ et ses différents emplois lexicalisées [even], qui pour un mot donné seront décrits sous la forme :

$$
[\% / \text { even } /]_{\mathrm{cat} S \mathrm{Sl}}
$$

dans laquelle / ../ est le morphème (et $\sigma$ sa signification ${ }^{16}$ ) tandis que $[. .$.$] est le$ lexème caractérisé par sa catégorie cat et son sens en emploi mais lexicalisé Sl (e.g. «plat», «calme », "régulier», « uniforme », etc. pour le seul adjectif even). Avec comme résultat de modifier aussi la représentation des bases des mots complexes quand celles-ci sont liées ou non-lexicales (e.g. encastrer, incruster).

S'agissant de signaler pour finir les forme les plus énigmatiques d'inconsistance sémantique que l'on peut parfois observer, - et qui pourraient sembler relever d'un manque absolu de rigueur scientifique ${ }^{17}$ si elles n'étaient pas techniquement indispensables pour escamoter le lapin sémantique - on observe sous les formes les plus diverses et des plumes variées une propension douteuse à la substitution anaphorique indue de syntagmes qui en réalité ne sont aucunement substituables.

Sans s'attarder sur les glissements qui reviennent à assimiler implicitement sens normal, ou sens fondamental et sens fixe, on assiste en effet depuis 40 ans à un tour de passe passe censé permettre de passer du constat (intuitionniste) de l'absence de sens fixe, ou d'une affirmation (péremptoire) sur le sens normal de quelque chose, à l'absence de sens ... d'autre chose puis à l'absence de sens tout court Comme quand ${ }^{18}$ décrivant l'hypothèse lexicaliste d'Aronoff à propos de remit, demit, transmit, transfer, refer, defer, prefer, Sergio Scalise et Emiliano Guevara $(2005,157)$ en viennent à passer sans crier gare de «no fixed meaning » à « without meaning » :

«A theory based on morphemes would have to recognize the stems fer and mit, and the prefixes re- de-, pre-, trans-. However, it is clear that in these examples «neither the suffix nor the stem has any fixed meaning 》 (Aronoff, 1976, 12). These units without meaning cannot be considered

16 Autrement dit la contrainte sémantique à satisfaire dans tous les emplois.

17 Ces raisonnements erratiques continuant à être repris à l'identique 40 ans après leur première formulation.

18 L'exemple cité ne peut être considéré comme déformant le raisonnement en question qui, répété de nombreuses fois, ne peut pas exister sans de tels glissements. Comme en témoigne dès 1976 l'utilisation de receive ou reduce par Aronoff pour disqualifier la description de re-par back. Démarche qui implique les mêmes glissements et le même postulat de l'existence d'un re-supra-sémantique. 
minimal linguistic signs (i.e. they are not Saussurean, arbitrary constant association of sound and meaning)" 19 .

Car ce qui rend le glissement non seulement indu mais parfaitement contradictoire est le fait même d'utiliser le syntagme « le suffixe $X$ » comme s'il existait un suffixe $X$, ce qui sémantiquement n'est acceptable que si $X$ est conçu comme polysémique, pour en conclure ensuite que ce que l'on devrait nommer " les suffixes homonymiques $X$ » n'ont eux aucun sens stable, ce qui n'est manifestement pas le cas. On voit que c'est finalement le seul fait de postuler l'existence d'un suffixe $X$ (ou d'une base ${ }^{20} X$ ) qui permet d'en conclure ensuite qu'il (ou elle) n'a pas de sens et qu'il (elle) n'est pas un signe saussurien, alors comme nous l'avons vu que si l'on écarte la polysémie on ne peut postuler l'existence que de plusieurs suffixes $X, X^{\prime}, X^{\prime}$ auxquels on ne peut dénier alors d'avoir des sens distincts et donc d'avoir des sens, y compris saussuriens.

Parce que parler d'absence de sens constant suppose de parler d'absence de sens constant de quelque chose mais que rien ne garantit l'unité de ce quelque chose, il est absolument inacceptable de dire que ce quelque chose n'est pas un signe saussurien. Un signe (même saussurien) doit d'abord être une unité, et il n'est pas possible de définir ce qui est une unité sur des bases non-sémantiques (et en refusant la polysémie) pour ensuite dire de cette unité qu'elle n'a pas de sens $^{21}$. A fortiori quand on prétend s'appuyer sur ce type de raisonnement pour en tirer des conclusions radicales sur la relation entre sens et forme ou pour écarter des descriptions somme toute assez efficaces. Et encore plus quand ce traitement n'est pas appliqué ensuite aux lexèmes, auquel aucun d'entre eux ne résisterait ${ }^{22}$.

19 Les parties en gras sont soulignées par moi.

20 Le fait d'employer des termes plus neutres comme "verbal element" et "thread/ fil"- comme dans « however, this verbal element has no identifiable meaning: that is, there is no common semantic thread linking assume, presume, resume and consume, such that the differences between them can be straightforwardly ascribed to the different prefixes. Therefore-sume cannot be regarded as a Saussurean sign, because it has no identifiable signifé." (Carstairs-MacCarthy 2006, 20) - ne change rien au fait que la conclusion consistera toujours à dire de lui (de cet élément) qu'il n'est pas un signe saussurien, sans plus rien dire des différents -sume, qui eux pourraient être décrits sans difficulté comme des signes saussuriens.

21 Il faut souligner que la sémantique linguistique a depuis longtemps établi que les sens sont liés à des emplois (récurrents) des signes, qu'il est absolument normal que ces sens diffèrent d'un emploi-type à l'autre et que la question de la constance ne se pose qu'en termes de satisfaction par chacun de ses sens d'une contrainte sémantique unique nommée signification, et qui n'est pas intuitive.

${ }^{22}$ La façon même de formuler habituellement la question - par exemple chez Andrew Carstairs-MacCarthy $(2006,7)$ "Do morphemes count as signs or do only words count, or both?" - trahit le fait que les tenants de l'hypothèse lexicaliste ne réalisent pas les 
De deux choses l'une en effet soit les morphologues pour ne pas parler au pluriel des suffixes re- ou -trans posent une hypothèse polysémique et il y aura par définition une forme de continuité sémantique entre les emplois décrits, soit ils refusent cette continuité et ne peuvent ni prédiquer quoi que ce soit d'un morphème qui n'existe pas ni escamoter en le faisant les morphèmes homonymes qui eux existent alors bien (ni a fortiori poser que ceux-ci ne sont pas des signes saussuriens). Quand, comme le reconnaît Aronoff à propos de $r e$ - en anglais et de sa paraphrase back, on a une interprétation sémantique qui « marche » pour énormément d'emplois verbaux de re-, on ne peut pas cinq lignes plus bas - après avoir mentionné deux cas pour lesquels cette paraphrase ne marcherait pas, à savoir reduce et receive, et fournit du premier un seul exemple - en conclure que le morphèmes ne sont pas des signes et n'ont pas de sens. De deux choses l'une en effet :

- soit il y a des raisons de supposer la présence de re-dans receive, et il faut alors les rendre explicite et s'assurer qu'elles permettent de postuler l'existence d'un unique morphème $r e$ - et alors, et seulement alors, il sera possible de faire les comparaisons avec les emplois glosables par back;

- soit il n'y a pas de critères sérieux ou encore il y a bien des critères mais ceux-ci excluent a priori l'existence d'un morphème unique et alors tel ou tel emploi de reduce ne pourra tout simplement pas constituer un contre-exemple aux centaines de tests positifs de la paraphrase back.

Ce qui est clair en tous les cas, c'est qu'il est scientifiquement inacceptable de produire des énoncés qui d'un segment de l'énoncé à l'autre adoptent les différentes positions, assumant ici l'existence d'un morphème pour immédiatement le nier ... tout en continuant à la supposer ensuite ${ }^{23}$. Et ce aussi bien pour des simples raisons de consistance logique que parce qu'on en vient sinon à quelque chose d'aussi absurde que d'écarter des centaines d'emplois de re- dotés d'une interprétation stable paraphrasable par back, sur la base du fait qu'un seul emploi de reduce ne vérifie pas ce test. Autrement dit,

conséquences du fait qu'aucun mot, y compris comme nous le verrons l'exemple saussurien de relire, ne résisterait aux critères de fixité du sens imposés aux morphèmes, à savoir en toute rigueur qu'il n'y a pas de signes du tout.

${ }^{23}$ Les seules solutions décentes pour reconnaître un constituant sont soit de postuler de la polysémie $-\left[{ }^{\sigma 1} / \mathrm{morph} /\right]_{\mathrm{S} 1}\left[{ }^{\sigma 1} / \mathrm{morph} /\right]_{\mathrm{S} 2}\left[{ }^{\sigma 1} / \mathrm{morph} /\right]_{\mathrm{S} 3}-$ soit de postuler de l'homonymie $\left[{ }^{\sigma 1} / \mathrm{morph} /\right]_{\mathrm{S} \alpha}\left[{ }^{\sigma 2} / \mathrm{morph} /\right]_{\mathrm{S} \beta}\left[{ }^{\sigma 3} / \mathrm{morph} /\right]_{\mathrm{S} \gamma}-$ soit de postuler une unité dénuée de signification - $\left[\backslash \text { cran } \backslash[\text { berry }]_{\mathrm{S} 1}\right]_{\mathrm{SDl}}$. Dans ce dernier cas, on ne peut néanmoins pas dire que l'élément concerné n'a pas de sens, puisqu'il acquiert en réalité obligatoirement une valeur distinctive (et par exemple la capacité de servir à désigner/dénommer un référent distinct), mais simplement que ce sens n'est pas obtenu à partir d'une signification comme dans tous les autres cas. 
juste après avoir plaidé la pluralité des sens, en conclure (Aronoff, 1976,4) une seconde plus tard qu'il ne reste plus «qu'une conclusion possible », à savoir que « il y a des morphèmes qui n'ont pas de sens ».

En somme, il serait donc raisonnable pour sortir de cette inconsistance :

- de s'interdire définitivement de postuler des d'unités sémantiques capables de n'avoir aucun sens et, simultanément, d'en avoir plusieurs, ces unités ne pouvant par définition pas exister ;

- de ne plus adopter en morphologie pour les morphèmes une position inverse de celle qui est adoptée en syntaxe pour les lexèmes, en refusant par principe l'homonymie en morphologie pour les morphèmes et en l'adoptant systématiquement en syntaxe pour les lexèmes ${ }^{24}$;

- de ne pas demander aux morphèmes ce qui n'est pas exigé des lexèmes ;

- d'accepter la polysémie comme une réalité sémantique incontournable et comme une hypothèse de travail raisonnable ;

- d'accepter la polysémie comme quelque chose de normal et dont il faut comprendre la normalité;

- d'abandonner la conception du signifié qui est associée chez Saussure à la définition du signe, cette conception étant manifestement incapable de rendre compte de la polysémie ;

- d'adopter une conception au moins benvenistienne du signe $[\sigma / \mathrm{m} /]_{\text {cat } \mathrm{s}}$, autrement dit une conception du signe capable de rendre compte de la polysémie.

Reste alors la nécessité, au-delà des glissements anaphoriques des tenants d'une « word-based morphology », de dissiper définitivement en morphologie une conception singulière du statut sémantique du contre-exemple qui est à la base du (déraisonnable) raisonnement d'Aronoff, et qui consiste à croire qu'un contre-exemple aurait le pouvoir assez étrange de faire disparaître empiriquement ce qu'il contredit. Une telle conception, pour le formuler clairement, confond en effet sous un même terme, à savoir sens, le sens comme hypothèse externe définissant ce dont il faut rendre compte (l'observable) et le sens comme hypothèse interne définissant la façon dont on en rend compte. Alors que l'immense mérite de Benveniste, et ce qui fait qu'adopter une conception benvenistienne du signe est en quelque sorte obligatoire en sémantique, est précisément d'avoir gardé le terme sens pour le premier et adopté celle de signification pour le second.

Qu'il y ait en effet en anglais beaucoup d'emplois de $r e$ - qui s'inscrivent

${ }^{24}$ Ces deux attitudes traduisant en réalité un refus commun d'affronter la question de la polysémie. 
dans une interprétation glosable par " again » est une réalité empirique incontournable mais aussi délimitable (par des tests) qu'aucun raisonnement ne peut annuler et qui s'impose au linguiste. Tout comme il y a en anglais beaucoup d'emplois de $r e$ - qui s'inscrivent dans une interprétation glosable par «back», ce qui est aussi une réalité empirique incontournable et délimitable par des tests et qui s'impose au linguiste. Moyennant quoi, aucune de ces deux interprétations de re- (ou, à ce stade, de ces deux re-), ne peut être considérée empiriquement comme un contre-exemple de l'autre, pas plus qu'aucune d'entre elles ne peut se voir nier en tant que réalité empirique.

S'agissant ensuite de savoir si l'une ou l'autre sont susceptibles d'être la signification de $r e-$, la question devient alors tout autre, car c'est là que commence en réalité le véritable travail du sémanticien, où si l'on préfère l'étape sémantique de tout analyse morphologique. Dès lors que c'est précisément le rôle de la sémantique de partir d'interprétations divergentes, comme celle de relire, pour établir s'il existe ou non une contrainte commune que chacune de ces interprétations satisferait. Constatant dans ce cadre que l'interprétation « de nouveau » n'est pas vérifiée par l'ensemble des emplois de re-, il/elle pourra en conclure d'emblée que « de nouveau » n'est pas la signification de $r e$ - qui par définition doit l'être, ceci sauf à postuler l'existence de plusieurs $r e-$, ce qu'il doit s'interdire de faire au moins dans un premier temps. Amené ensuite à interroger une contrainte partagée par les emplois en «again » et " back », par une démarche qu'il n'est possible ici que d'évoquer, il/elle constatera qu'il est en réalité facile de subsumer « again » à « back », dès lors que toute situation décrite par le premier implique une étape qui fait partie de toute situation décrite par «back», par exemple comme nous le verrons du fait que « il repleut » comme « il revient sur ses pas » impliquent l'un et l'autre un retour à une situation ou position antérieure. Et c'est ce constat, le plus souvent corrélé au remplacement de tests paraphrastiques par des tests déclaratifs, qui lui permettra d'abandonner l'hypothèse d'une homonymie au profit d'une explication polysémique permettant de démontrer que les deux interprétations, qui peuvent être cumulables sur des emplois dits "pont», ne sont que des formes différentes de satisfaction d'une contrainte unique. Il passera alors au constat que d'autres emplois encore existent qui dans l'hypothèse d'une non homonymie doivent être expliqués, et que ces emplois peuvent constituer - à ce stade de formulation de la signification - d'authentiques contre-exemples, à charge pour lui de comprendre très exactement ce en quoi ils divergent des cas traités antérieurement. L'existence de contre-exemples ne justifie donc ni de jeter au rebut des descriptions déjà très efficaces (et a fortiori les données qui les soutiennent) ni d'être utilisée comme prétexte pour arrêter toute sémantique : le travail du sémanticien consiste à progressivement intégrer la plus grande 
diversité possible d'emplois dans une explication unique (si celle-ci existe), travail qui va par définition lui prendre plusieurs semaines s'il veut comprendre en détail la totalité des formes de variation de l'interprétation. Ce qui au total interdit de croire qu'un contre exemple puisse ruiner une description de la signification, cet exemple ne pouvant que prouver que celle-ci ne marche par exemple que dans $90 \%$ des emplois observables, ni justifier de l'abandonner et avec elle les $90 \%$ de données concernées : le statut méthodologique du contreexemple est donc uniquement de mettre le sémanticien dans l'obligation de devoir soit intégrer l'exemple concerné soit en faire un homonyme. Moyennant quoi, la seule démarche scientifiquement légitime en matière de sémantique est d'interroger le sémanticien sur la possibilité de partage d'une contrainte entre ce qui pourrait être différents emplois d'un même signe et de lui donner le temps nécessaire pour y répondre. A l'opposé de la croyance selon laquelle il suffit de brandir un seul contre-exemple pour jeter une explication efficace avec l'eau du bain, il faut en effet moins d'une heure à un sémanticien spécialiste de polysémie ${ }^{25}$ pour montrer que la distance entre l'interprétation des emplois de reduce et de receive et les emplois en back est mineure, ce qui certes condamne d'une certaine façon la glose en back - sans nier qu'elle soit bien sinon la signification du moins une interprétation-type largement partagée (et par ailleurs non intuitive) - mais ne remet en cause ni la polysémie (qui s'avère seulement plus large encore) ni l'unité du signe. Car s'il est exact que du point de vue sémantique on ne comprend souvent pas initialement grandchose à l'observable, il est absolument déraisonnable, en particulier si c'est à la seule fin de défendre la thèse qu'il n'y a rien à comprendre, de poser le fait de tout comprendre immédiatement comme un pré-requis indispensable aux explications proposées : la capacité de prédiction de la sémantique d'une unité étant une fonction quasi-linéaire du temps qui lui a été consacré, l'intérêt de toute description doit être mesuré autant en termes de ce qu'elle permet déjà d'unifier qu'en termes de ce qu'elle ne permet pas encore d'unifier. Surtout dès lors que si rien ne justifie jamais d'ignorer le premier aspect de la question, on constate que reprocher à une explication de ne pas toujours marcher, va presque toujours de pair avec la défense d'explications ayant une capacité de prédiction inférieure mais qui sont intuitives (de type «the meaning of $r e$-is approximately again $\left.»^{26}\right)$. Ce qui revient à dire que pour ce qui est de rendre compte de la

25 On sait en effet depuis au moins Sapir (1944) que l'interprétation des expressions de quantité donne lieu à des lectures très spécifiques. Réduire concernant très souvent une orientation à la baisse de quelque chose qui se situait à un niveau plus élevé, on comprend aisément que la glose back ne fonctionne pas, alors que le mouvement concerné est au bout du compte identique. Ce qui impose de décrire les gloses backwards ou downwards comme des profils.

${ }^{26}$ On arrive alors à ce paradoxe extraordinaire pour une démarche censée relever de 
diversité des emplois, le seul critère qui compte est en quelque sorte le taux d'élucidation et le taux de couverture et que toute comparaison de ce point de vue doit rester quantitative. S'agissant de $r e$-, il faut constater que la glose en back représentait en 1973 un progrès considérable et qu'il est regrettable qu'au lieu d'interroger son efficacité relative et d'en tirer des enseignements utiles sur le rapport forme/interprétation, elle soit restée ignorée au profit de conceptions farfelues de la nature des phénomènes interprétatifs.

Comme nous l'avons vu à propos de la polycatégorialité, l'idée héritée de la définition structuraliste du morphème selon laquelle les morphèmes sont à la fois les briques combinatoires de la langue (puisque obtenus par décomposition) et les briques sémantiques de la langue, ne résiste pas au constat que les morphèmes sont des unités non-catégorielles qui peuvent être insérés dans plusieurs positions grammaticales (et ce sans ${ }^{27}$ qu'il y ait conversion d'une catégorie à l'autre ${ }^{28}$ ). Cette réalité implique en revanche d'adopter une définition purement distributionnelle du morphème comme paire forme ${ }^{29} /$

l'activité scientifique que l'analyse sémantique doive alors avant tout pouvoir conforter l'intuition immédiate et qu'à ce titre toute sémantique qui demanderait un peu de temps soit par avance exclue. J'ai eu l'occasion de nommer « the five-minutes semantics fallacy » ce présupposé consistant à poser péremptoirement des affirmations de type «there is no way this meaning could be related to this meaning », sans même avoir, ne serait-ce qu'une seule seconde, essayer de voir si cela était le cas ou non.

27 Tous les travaux sur la polycatégorialité montrent que la signification n'est pas catégorielle, contrairement au sens qui inclue une interprétation catégorielle de la signification. L'explication technique de cette réalité est que l'indication morphémique codée est au moins de la taille d'une phrase énoncée. L'explication sémantique est que l'interprétation est un processus de type fractal (Robert, 2003), chaque élément de la phrase énoncée ayant une signification d'une taille au moins égale à celle d'une phrase. Ce qui formellement veut dire qu'au lieu d'avoir par exemple des formes logiques russellienne de type « $a R b$ » on aura des formes logiques fractales de type $\left(\mathrm{cR}_{2} \mathrm{~d}\right)_{\mathrm{a}=\mathrm{c}} \mathrm{R}_{1}\left(\mathrm{eR}_{3} \mathrm{f}\right)_{\mathrm{b}=\mathrm{f}}$. Le fait que $a$ soit un nom n'implique donc jamais que la signification du signe employé pour nommer $a$ doive être nominale (Cadiot \& Nemo, 1997b) et explique l'échec de toutes les paraphrases nominales à la décrire. On voit la distance considérable qui existe entre la représentation du lexique qui est celle d'un dictionnaire, utilisant paraphrases et équivalents constructionnels (e.g. « un meuble est un objet...») et une représentation sémantique qui pour être explicative devra ignorer ces paraphrases et chercher à identifier pour l'adjectif meuble comme pour le nom meuble, le scénario encodé par le morphème, en l'espèce identique à celui de mobile (ce qui explique mobilier, etc.), scénario qu'il faudra interpréter dans chaque emploi (e.g. un sol meuble).

${ }^{28}$ Ce qui ne signifie pas qu'il n'y ait pas de conversion d'une catégorie à l'autre, puisqu'on peut parfaitement avoir des objets morphologiques ayant la forme $\left[[\mathrm{lex}]_{\text {Cat1 }}\right]_{\text {Cat2 }}$. Pour décrire un lexique non catégoriel comme pour décrire la polycatégorialité, les unités lexicales auront néanmoins toujours une forme de type $[\% / \mathrm{m} /]_{\mathrm{Cat} 1 / \mathrm{Sl}}$.

${ }^{29} \mathrm{La}$ forme étant elle-même flexible comme le montre notamment les deux linéarisations de forme en français, à savoir /form/ et /morf/. L'important étant de démontrer l'existence d'un sigma unique ${ }^{\sigma 1} /$ form/ et ${ }^{\sigma 1} /$ morf/. 
signification indépendante du contexte d'insertion et de ne plus utiliser pour le coup l'opposition morphème libre/morphème lié, le /table/ du nom $\left[{ }^{\sigma} / \text { table/ }\right]_{\mathrm{N} / S \mathrm{I}}$ étant, et ce de façon sémantiquement prouvable ${ }^{30}$, le même morphème que le / table/ que l'on observe dans les verbes $[\sigma / t a b l e /-\mathrm{er}]_{\mathrm{V}}$ ou $[\% / \text { ré/- } \sigma / t a b l /-\mathrm{ir}]_{\mathrm{V}}$. Il faut souligner que cette définition recoupe et conforte paradoxalement la réticence à faire des morphèmes des unités grammaticales de base, en dissociant la question des unités sémantiques de base de la question des unités grammaticales de base.

Doit donc aussi être abandonnée l'idée selon laquelle on ne peut reconnaître la présence d'un morphème dans un mot que si l'on est capable de décomposer exhaustivement ce mot. Et ce précisément, et comme nous allons le voir à propos de $r e-$, dans la mesure où l'approche distributionnelle du morphème permet d'isoler sa signification sigma sur des exemples où le découpage n'est pas problématique et de vérifier ensuite sur les exemples problématiques si cette contrainte sémantique sigma est satisfaite. Il est alors possible de reconnaître la présence d'un morphème dans des mots sans avoir à théoriser immédiatement l'ensemble des constructions dans lesquelles il se trouve inséré/ utilisé $^{31}, \mathrm{y}$ compris quand certains mots s'avèrent comporter des segments «non significatifs » mais qui n'interdisent en rien l'interprétation des autres éléments.

Reste à signaler une dernière conséquence d'une définition acatégorielle/ distributionnelle du morphème, qui n'a émergé que récemment en sémantique de la signification, à savoir de permettre de résister à la tentation de mettre dans la signification d'un morphème la sémantique de la construction dans laquelle il est inséré. Ceci sachant que c'est bien l'idée inverse qui a longtemps prévalu, et ce aussi bien : i) en sémantique instructionnelle chez Oswald Ducrot quand on constate que décrire l'instruction de mais revient à décrire ce que le morphème dit de l'enchainement « p mais q »; ii) en morphologie générative quand on a voulu mettre dans les règles sémantiques associées aux affixes une description du rapport sémantique supposé entre input supposé et output constaté (méthode soustractive) ; iii) en morphologie constructionnelle quand Danielle et Pierre Corbin dans le traitement de -ier (Corbin \& Corbin, 1991) adoptent une démarche instructionnelle mais conçoivent in fine l'instruction

30 Sémantiquement prouvable signifie ici d'une part qu'il n'y a pas héritage lexical du/ d'un sens S1 du nom [table], et donc pas dérivation, et d'autre part qu'il y a bien satisfaction de la contrainte sémantique sémantique sigma (cf. sur ce dernier point Nemo, 1999 et Nemo, 2004, 17)

${ }^{31}$ Ce qui signifie pour en rester encore ici à une valeur d'emploi de $r e$ - qui n'est pas sa signification sigma, à savoir la valeur de répétition (i.e. de nouveau, deux fois, une nouvelle fois, etc.), que l'on peut reconnaître la satisfaction de cette valeur dans des mots comme répétition récurrence ou redondance sans avoir besoin pour le faire de justifier préalablement de la nature des autres composants ou de trancher la question du caractère régulier ou non des constructions considérées. 
comme décrivant la construction ; iv) en grammaire de construction quand Adèle Goldberg (1995) en vient à dire que le morphème est une construction parce qu'il est une paire forme/sens ${ }^{32}$. Et qu'il a donc fallu attendre la description de morphèmes à distribution polycatégorielle ${ }^{33}$ comme encore, enfin ou tout/toujours pour qu'apparaisse radicalement ${ }^{34}$ la nécessité de poser que la signification d'un morphème ne préfigure jamais son utilisation constructionnelle (Nemo, 2009) et qu'il faut en fait se servir de la diversité des constructions dans lesquelles un morphème est inséré pour précisément séparer les contraintes sémantiques imposées par le morphème de celles qui sont imposées par les constructions.

Plus globalement, ce qu'il faut comprendre, c'est qu'en matière de sémantique, qu'il s'agisse de morphologie ou de syntaxe, il est souhaitable de ne pas postuler a priori l'existence de rapports forme/sens stables, et ce aussi bien au niveau des unités (morphèmes ou lexèmes) qu'au niveau des constructions, et au contraire de se donner comme objet fondamental d'expliquer l'instabilité du rapport forme/sens d'une part et l'ensemble des formes de stabilisation de ce rapport d'autre part. Faute de quoi, comme nous allons le voir maintenant à propos des listèmes (Di Sciullo \& Williams, 1987), la focalisation sur les formes de stabilisation du rapport forme/sens se paie inévitablement par la mise à la poubelle ${ }^{35}$ de la plus grande partie du lexique, Réalité qui va nous conduire à nous tourner maintenant vers les listèmes, en tant que ceux-ci sont précisément

32 Les bases logiques de cette équivalence peuvent elles aussi laisser pantois. En réalité, un morphème est en quelque sorte l'inverse d'une construction, puisqu'il est une paire forme/ signification indépendante du contexte d'insertion alors que la construction est une paire forme/sens indépendante du matériau sémantique utilisé. D'où l'importance cruciale en sémantique d'utiliser en parallèle les deux techniques et de comprendre leur relation, les lexèmes étant (minimalement) des paires morphème/construction.

${ }^{33}$ Contrairement à celle de mais, qui est strictement connective.

34 On peut considérer que dès 1980, le traitement réservé par Ducrot (1980) aux emplois de mais qui ne satisfaisaient manifestement pas la verbalisation associée à la description instructionnelle de la construction argumentative « $\mathrm{p}$ mais $\mathrm{q} »-$ traitement qui consistait en substance à montrer que même si la glose constructionnelle ne fonctionne pas en tant que telle, il y a bien quelque chose de très proche qui lui fonctionne - avait montré que les formulations de l'instruction sémantique tendent systématiquement à être trop spécifiques et trop constructionnelles. L'étape suivante a donc consisté à ne plus chercher à décrire simultanément le morphème et les constructions.

35 Le terme poubelle est employé ici en référence au pragmatic wastebasket de BarHillel (1971). La situation est à bien des égards pire en morphologie dès lors qu'il ne s'agit pas seulement pour un modèle de se débarrasser des données qui le falsifient, en l'occurrence de sauver une mauvaise sémantique en invoquant une discipline alors inexistante, mais bien comme nous l'avons vu de jeter celles-ci définitivement aux oubliettes. Il existe néanmoins et dès le milieu des années 70 chez Danielle Corbin par exemple, des morphologies capable de s'interroger sur le statut réel des « exceptions » supposées. 
les traces de l'instabilité du rapport forme/sens, et ce pour interroger le rôle de l'interprétabilité dans la question de la formation des mots. Ce que nous ferons en étudiant un ensemble de listèmes ayant en commun la présence en leur sein du morphème $r e-$, présence qui peut être mieux comprise à partir de :

- la double caractérisation de la signification morphémique comme déclencheur du processus interprétatif et contrainte à satisfaire et du sens lexical comme aboutissement de ce processus et comme mémoire des différentes formes de satisfaction de ces contraintes (Nemo, 2002b).

- la redéfinition des constructions sémantiques comme paires routine interprétative/structure et comme contraintes secondaires (Nemo, 2010), autrement dit par une clarification de la relation entre instruction sigma et construction.

\section{LES LISTÈMES}

On appelle listèmes depuis Di Sciullo \& Williams (1987) tous les items lexicaux qui ne sont pas prédictibles à un titre ou à un autre (grammaire, sens, forme, constituants) et dont on suppose non seulement qu'ils doivent être appris un par un mais qu'ils ne méritent pas de ce fait d'être étudiés ${ }^{36}$.

Moyennant quoi, le terme renverra aussi bien aux lexèmes «atomiques» (non complexes non structurés non construits pour employer la terminologie de Danielle Corbin) qu' aux lexèmes complexes non prédictibles ${ }^{37}$ pour une raison ou une autre.

\subsection{LES CAUSES DE LISTAGE}

Les causes de listage constituent en fait le miroir exact des présupposés des modèles impliqués, ces présupposés étant qu'un mot bien formé est toujours obtenu à partir d'un autre mot (thèse dérivationnelle ou compositionnelle), qu'il existe un mécanisme combinatoire régulier permettant d'obtenir le second à partir du premier, qu'il existe un rapport sémantique stable entre le sens de l'input et le sens de l'output et que l'ensemble des constituants sont non problématiques;

Moyennant quoi sont donc automatiquement listés les mots qui n'incluent pas sémantiquement de mots (.e.g. empêtrer, représailles, obstacle), les

36 Le raisonnement existait déjà chez Aronoff (1976) quand il entendait distinguer deux classes de mots, ceux dont le rapport forme/sens était trop idiosyncrasique pour pouvoir relever d'une règle générale et ceux qui étaient crées par la mise en œuvre de cette règle supposée.

37 Je n'emploie et n'emploierai ici le terme que pour ces derniers. 
mots associant un input sémantiquement transparent mais non attendu catégoriellement, e.g. chimiquier à un output sémantiquement et catégoriellement prédictible mais, les mots pour lesquels le sens de l'input potentiel apparaît non pertinent (par exemple dé-valis-er ou dé-rob-er, ré-tabl-ir ou rot-ation) et les mots pour lesquels est postulée une « dérive » sémantique entre input et output (e.g. l'avion décolle, etc.). Ce qui au bout du compte, en particulier du fait des phénomènes dits de "dérive sémantique », fait que la plus grande partie du lexique est constituée de listèmes, au point que les promoteurs déjà cités de la notion en sont venus à décrire le lexique comme lieu de l'irrégulier, ou encore comme « une prison dont les occupants n'ont en commun que de ne respecter aucune loi », et qui à ce titre ne méritent pas d'êtres étudiés, créant ainsi un «morphological wastebasket ». Le tout sachant que les choses seraient bien pires si au lieu de lister des mots, objets qui en réalité n'ont aucune sorte d'existence sémantique, on classait les emplois lexicalisés des dits mots, la plus grande partie des emplois des mots non listés s'avérant non prédictibles, comme nous le verrons à propos de relire.

\subsection{QUELQUES LISTÈMES INCLUANT LE MORPHÈME RE-}

S'agissant aussi bien d'interroger la capacité d'une sémantique générale à rendre compte d'un ensemble de listèmes et d'interroger ce que cet ensemble a à nous dire en ce qui concerne l'interface entre sémantique et morphologie, nous allons nous donner comme objectif d'expliquer et d'analyser les listèmes suivants :

- représailles, rétorsion, revanche, réprouver, reprocher, remontrance, réprimander, répréhensible, etc.

- répondre, répliquer, rétorquer, riposter, etc.

- regimber, renâcler, refuser, répugner à, rechigner, répulsion, rébarbatif, récalcitrant, se rebeller, réfractaire, rétif, réticence, rebuffade, se rebiffer, révulser, se récrier, résister, etc.

- refléter, réfléchir, réverbération, etc.

- renier, renoncer, reculer, etc.

- retenir (un texte), revenir (sur ses propos), refouler (une émotion), etc.

- réciproque, etc.

et ce à la fois du point de vue de leur interprétation que de celle de la présence du signe $r e$ - lui-même. 


\subsection{LE LISTAGE COMME COÛT THÉORIQUE ET ARTEFACT THÉORIQUE}

Le coût théorique d'un modèle (Ducrot $\&$ al, 1980) se mesure à la réduction de l'observable par la théorie avancée, autrement dit à la façon dont les hypothèses externes (définissant ce dont il s'agit de rendre compte) se trouvent en réalité sélectionnées par les hypothèses internes (le type d'explication mis en œuvre). On peut de ce point de vue considérer le listage de la plus grande partie du lexique comme le prix que certaines morphologies acceptent de payer pour sauver leurs conceptions de la nature des mécanismes sémantiques ou linguistiques, qui en l'espèce seraient erronées. Et on peut aussi considérer que parler de coût théorique n'est qu'une façon euphémisée de dire que chaque listème étant une falsification des modèles de type WFR ou dérivation, et/ou des hypothèses sémantiques ou méthodologiques de celles-ci, c'est bien ces modèles et non les listèmes en question qui devraient être profondément reconsidérés. Ce qui pour le dire encore autrement consiste à poser qu'en matière de lexique, il est peut-être temps de ne plus décrire seulement la partie émergée de l'iceberg (i.e. la fraction du lexique accessible à l'intuition immédiate) et d'interroger les mécanismes qui rendent totalement non-problématiques car interprétables des listèmes comme rotation ou amorphe et la plupart des emplois de non-listèmes supposés comme relire.

\subsection{LE LISTAGE COMME IMPASSE THÉORIQUE}

Mais si le listage est véritablement une impasse, c'est comme nous allons le voir maintenant pour une raison bien plus importante, à savoir le fait que l'on peut prouver qu' il est en pratique impossible de rendre compte des non-listèmes sans rendre compte des listèmes et par exemple impossible de rendre compte d'un non-listème apparent comme relire sans rendre compte de l'ensemble des listèmes que nous venons d'introduire. Ceci dans la mesure où si l'on prend des mots non listés car apparemment non problématiques, force est de constater que tout modèle capable de rendre compte des sens de relire ou de la différence d'interprétation entre déterrer et désherber (Nemo, 2010), sera aussi capable d'expliquer les problèmes qui conduisent au listage.

\subsubsection{Sémantique rapide de relire}

Car si rendre compte de la forme linguistique de relire n'a rien de complexe ${ }^{38}$, expliquer ses sens est en réalité une toute autre affaire. D'abord parce que relire a au moins 3 emplois courants en français, et non un comme le disait Saussure, et qui correspondent à au moins trois situations distinctes,

38 Dans la mesure où rien dans la structure de $\left[\text { re- }[\text { lire }]_{\mathrm{V}}\right]_{\mathrm{N}}$ ne pose de problème et que sémantiquement son interprétation est intuitive. 
à savoir des situations où on va soit (re)lire quelque chose que l'on avait déjà lu (c'est le sens le plus intuitif), soit (re)lire ce que l'on vient d'écrire (par exemple sa copie ou son powerpoint), soit (re)lire ce que quelqu'un d'autre a écrit. Et ensuite parce ce que ces trois interprétations ne sont pas hiérarchisables entre elles, du fait notamment qu'aucune d'elles - sauf à faire de l'intuition immédiate du sujet parlant la boussole du linguiste, ce qui scientifiquement est inacceptable dans quelque domaine que ce soit $^{39}$ - ne peut prétendre être « le sens linguistique de relire » ou «le sens construit de relire ». Et du fait que la moins intuitive des trois s'avère être la seule interprétation pertinente pour ce qui est de rendre compte du mot $"$ relecteur $»^{40}$. Le tout illustrant le fait que si rendre compte du sens linguistique, c'est rendre compte des contraintes linguistiques qui devront être respectées dans l'interprétation, il faut commencer par reconnaître qu'il y a le plus souvent plusieurs façons de les satisfaire ${ }^{4 l}$.

Moyennant quoi au lieu de penser que le morphème re-doit apporter une information précisant ou modifiant le sens de lire, on découvre alors (Nemo, 2002b, 2010) que la contrainte sémantique codée par re- (et satisfaite de façon différente dans les trois interprétations) est l'indication ${ }^{42}$ de l'existence de deux processus $\mathrm{p} 1$ et $\mathrm{p} 2$ anti-orientés ${ }^{43}$. Et qu'il est demandé à l'interprétant de chercher dans le co-texte ou le contexte d'identifier la nature exacte de ces deux processus et ce en quoi ils sont anti-orientés. On observe alors que dans les trois interprétations de relire, lire s'unifie avec le second processus p2 ${ }^{44}$; que dans

39 La sémantique n'est pas plus une théorie de l'intuition sémantique des sujets parlants que la syntaxe n'a vocation à l'être en ce qui concerne les idées que les sujets parlants pourraient avoir à propos de grammaire.

40 Le fait qu'un francophone ne soit pas capable de rendre compte du sens en emploi de amorphe en français ne devrait pas conduire à le considérer comme un listème dont il n'y aurait pas à rendre compte : il suffit de mettre en relation morph dans amorphe avec le sens de forme, qui s'observe dans «être en forme », « avoir la forme », «Décathlon à fond la forme » pour le rendre totalement prédictible. Là encore, toute approche se donnant la conscience sémantique comme boussole sombre dans un subjectivisme inavoué et radical et se condamne.

${ }^{41}$ Le fait qu'une même contrainte puisse être satisfaite de différentes façons définit la notion de polysémie.

${ }^{42}$ Cette double réalité correspond à la définition indicationnelle-indexicale (Nemo, 2001a) de la notion d'instruction sémantique introduite en sémantique depuis le début des années 80 (Ducrot \& al, 1980).

${ }^{43}$ Ce qui illustre le fait que « la signification est d'une autre nature que le sens » et qu'elle ne correspond jamais à l'intuition sémantique des sujets parlants, le sens de répétition s'avèrant n'être qu'une des façons de satisfaire la contrainte d'anti-orientation.

${ }^{44}$ Cette réalité qui n'a rien d'obligatoire demeure néanmoins la routine interprétative la plus fréquente en français, on la retrouve dans « repousser l'attaque » ou pousser est p2 
deux interprétations le processus p1 est « écrire » alors que dans le troisième il s'unifie avec «lire », l'anti-orientation concernant dans tous les cas le fait qu'arrivé au point d'arrivée de $\mathrm{p} 1$, il faut pour réaliser $\mathrm{p} 2$ retourner au point de départ de pl. Ce qui implique que lire est sémantiquement un argument (par ailleurs non obligatoire) du morphème $r e^{-45}$, que p1 n'étant presque jamais fourni dans la construction elle-même (l'un des rares contre-exemples en français étant la construction re: salut ! des courriers électroniques) sa nature sera variable et déterminée en mobilisant le contexte, mais que le fait de trouver un p1 anti-orienté à p2 sera une contrainte linguistique.

\subsubsection{Unités de l'ensemble des emplois de re et contrainte morphémique}

L'une des implications majeures de ce qui précède est que le linguiste doit éviter des gloses paraphrastiques censées lui fournir le sens d'un mot, mais qui décrivent avec une précision insuffisante ce qui est dit et s'avèrent non transposables aux autres emplois du morphème et inutilisables pour retrouver la constante morphémique sous la variabilité de l'interprétation. Ainsi dans «il repleut » comme dans beaucoup d'emplois de récurrence, l'enjeu morphémique n'est pas qu'il pleuve deux fois mais qu'un processus p1 (arrêt de la pluie) dont le point de départ est un état pluvieux et le point d'arrivée un état non pluvieux soit suivi d'un processus p2 avec comme point de départ cet état non pluvieux et point d'arrivée le point de départ de p1. Ainsi, dans réapparaît, la récurrence ne concernera souvent pas la répétition (i.e. «apparaître deux fois » ou « apparaître une nouvelle fois ») mais bien un processus inversant les points de départ et d'arrivée d'une disparition précédente $\mathrm{p} 1^{46}$.

Moyennant quoi, malgré les apparences, «il repleut» et «il est retourné chez lui » racontent en réalité très exactement la même chose et le même type d'histoire, qui est celle d'un retour au point de départ, ce qui fait que

est attaquer est p1. Dans l'emploi 2.0 du morphème re, celui des messageries électroniques (re : salut!), on constate à l'inverse que s'il y a toujours bien un p1 (le premier mail) et un p2 (le second mail) anti-orientés par le fait que l'émetteur devient destinataire et inversement, la construction n'est pas de type |re-p2| mais bien de type |re-p1|.

45 Et en ce sens, ce n'est pas re- qui précise le sens de lire mais bien l'inverse, lire fournissant un des éléments que la signification du morphème impose de rechercher.

46 Il ne faut donc pas confondre la question de l'unification morphémique et celle des paraphrases du sens obtenu. Dans beaucoup de cas de récurrence, on a ainsi une succession pA pB pA dont la glose est habituellement centrée sur la répétition de pA et masque donc la présence de $\mathrm{pB}$ (et donc le lien avec la glose «back »). Ce que montre par exemple le fait que l'interprétation de «j'ai repetitdéjeuné » ne renverra pas à la répétition quotidienne du petit-déjeuner, mais au fait par exemple d'avoir pris deux petits-déjeuners le même jour, ou d'alterner les jours (ou périodes) avec petit-déjeuner et les jours sans. 
l'interprétation itérative ${ }^{47}$ de re n'est en réalité que ce que les sémanticiens depuis Cadiot \& Visetti (2001) nomment un «profilage » sémantique de la signification morphémique.

Il importe dès lors pour le linguiste de ne pas jouer les interprétations les unes contre les autres, car si les profilages pour ne pas être la signification du morphème n'en sont pas moins des réalités empiriques qui ne méritent aucun mépris et qui doivent être décrits, ils ne sont cependant pas plus que cela et jamais plus centraux ou normaux les uns que les autres. Raison pour laquelle, la diversité des profilages interprétatifs ne doit pas comme nous l'avons vu être utilisée pour dénier aux signes leur statut sémantique d'unités imposant des contraintes sémantiques à satisfaire ou pour poser que les morphèmes n'ont pas de sens et ne sont pas des signes saussuriens. Ne jamais jouer les différentes façons ${ }^{48}$ de satisfaire une même contrainte sémantique les unes contre les autres apparaît comme d'autant plus indispensable que cela permet pour le coup de rendre compte à la fois théoriquement de l'instabilité du rapport forme/sens (et de la nature de l'interface entre sémantique et morphologie), mais aussi empiriquement de la plupart des listèmes. Le paradoxe étant en effet que loin d'être totalement imprédictibles et de ne respecter aucune loi, il est possible de montrer que les listèmes satisfont en réalité pleinement les contraintes sémantiques associées ${ }^{49}$ aux morphèmes employés, et par exemple qu'une fois identifiée la contrainte codée par le morphème $r$ - à partir de la polysémie de mots comme relire ou repousser ou revenir, on peut revenir aux listèmes pour constater que chacun d'entre eux (et/ou de leurs sens) satisfait bien cette contrainte. Dans l'idée de représailles ou de rétorsion, il y a bien en effet le présupposé $(=\mathrm{p} 1)$ que quelque chose de négatif pour quelqu'un s'est produit du fait de quelqu'un d'autre et qu'un second mouvement (toujours négatif) se produit ensuite avec inversion des rôles entre les personnes impliquées ${ }^{50}$. Ce

47 Qui pour être le sens le plus accessible de $r e$ - à l'intuition des sujets parlants (hors contexte) n'est donc qu'une des nombreuses interprétations possibles de re- (et aucunement son sens premier, rebondir ne voulant pas dire bondir deux fois et l'interprétation itérative étant très minoritaire dans les emplois de $r e$ ).

48 Le profilage en question est en effet utile, y compris en français, à la fois pour tester et éclairer la sémantique de certains listèmes comme reculer (test positif pour to move back) ou rendre (test positif pour to give back), et pour discriminer les différents sens d'un mot, par exemple en opposant les emplois où repousser est interprété comme «to push back» et les emplois où il ne l'est pas (e.g. "ses cheveux ont repoussé »).

49 Une contrainte est dite codée si on peut montrer qu'elle est satisfaite dans tous les emplois d'un signe, à la manière où un ensemble de points par ailleurs différents peuvent satisfaire une même équation.

${ }^{50} \mathrm{Ce}$ que l'on pourrait montrer aussi en anglais pour retaliation ou retorsion, parfaitement prédictibles eux aussi à partir d'une glose comme «to hit back», et incluant pour le premier une référence à la loi du talion. 
que l'on retrouve bien évidemment avec une notion comme celle de revanche, avec un passage de $\mathrm{p} 1$ (X l'emporte sur Y) à p2 (Y l'emporte sur X) montrant la même inversion des rôles sémantiques. S'agissant ensuite de réprouver, reprocher, remontrance, réprimander, on n'observe plus l'identité entre p1 et p2 propre aux exemples précédents, mais bien une forme d'opposition verbale ou morale au comportement antérieur d'une personne, répréhensible, en évoquant une punition comme conséquence d'un acte délictueux ou indélicat restant néanmoins plus proche de l'idée que quelqu' un qui fait quelque chose de mal (dont pâtit quelqu'un) doit à son tour subir quelque chose de désagréable. Dans les situations à propos desquelles sont employés les mots réticent, rétif, récalcitrant, refuser, renâcler, regimber, répugner, rechigner à, se rebiffer, se rebeller etc. on aura de même toujours de la part de quelqu'un une opposition $(=\mathrm{p} 2)$ à faire quelque chose qui lui a été demandé ou imposé $(=\mathrm{p} 1)^{51}$. Et il en est de même pour répondre, rétorquer, répliquer, riposter dont la sémantique suppose elle aussi un premier discours puis un second avec permutation des positions énonciatives et des orientations argumentatives, puisqu'à chaque fois le sujet de p2 (et du verbe) est émetteur d'un discours alors qu'il était récepteur d'un discours $\mathrm{p} 1$ antérieur, auquel il s'oppose dans les trois derniers cas ${ }^{52}$ mais aussi dans plusieurs emplois de répondre. Enfin, pour « surface réfléchissante », «parois qui réverbèrent la chaleur» et autres «carreaux de faïence qui reflètent les rayons du soleil », on a toujours quelque chose qui atteint une surface et que cette surface renvoie, et donc bien les mêmes mouvements p1 et p2 anti-orientés que l'on peut observer pour le non-listème rebondir.

La même chose pouvant être démontré pour chacun des autres mots de notre liste, qu'il s'agisse de reculer (to move backward) de la bi-directionalité de réciproque, ou de l'ensemble des emplois de retenir ou personne ne tient

51 On retrouve ce profil dans l'interprétation habituelle de « avancer à reculons », qui désigne bien toute l'ambivalence d'un mouvement p1 (avancer) contrarié par une volonté inverse. La seule différence entre ce type d'emploi et les précédents tient à ce que l'on appelle le profilage temporel de l'interprétation, qui concerne ici la relation temporelle entre $\mathrm{p} 1$ et $\mathrm{p} 2$ : si $\mathrm{p} 1$ précède souvent $\mathrm{p} 2$ dans les emplois de re-, ce profilage n'est pas codé et on observe fréquemment que p1 et p2 sont concomitants ou atemporels, comme dans les emplois de type «on se retrouve aux Deux Magots » ou encore «les rues de Vaugirard et de Rennes se rejoignent au niveau du numéro 54», dans lesquels pour le premier l'antiorientation ne suppose pas que le point d'arrivée de p1 soit le point de départ de p2 comme dans la successivité mais seulement la concomitance d'orientations à la fois distinctes et convergentes vers un même point d'arrivée.

52 Il faut noter que la polysémie des emplois de riposter (e.g. les emplois militaires et discursifs), indépendamment de la dimension lakovienne de la question («conversation is war») laisse inchangé et le profilage temporel, p1 étant antérieur à p2, et la similarité entre p1 et p2, et l'inversion des rôles. 
personne $e^{53}$, on ne peut que constater à quel point la sémantique de mots largement ou parfaitement opaques par ailleurs est en réalité aussi totalement prédictible - en ce qui concerne l'existence de deux processus anti-orientés que contextuelle quand à la nature de ceux-ci et de la façon dont ils s'opposent. Moyennant quoi, ce que l'on observe est donc que pour chacun de ces pseudolistèmes, et même de leurs emplois, il y a bien interprétabilité du morphème re- et satisfaction des contraintes qu'il encode. Et que de ce point de vue, qui est celui de l'interprétabilité de chacun de ces mots (et de leurs emplois), la seule différence entre ceux-ci et les mots considérés comme non listables, tient simplement au fait que contrairement à ceux-ci, l'interprétant ne trouve pas dans le mot lui-même le processus p2 qu'il cherche et doit le trouver dans le cotexte ou le contexte. Ce qui n'interdit pas pour autant à ces mots de demeurer parfaitement interprétables, parfaitement interprétés et surtout d'être sémantiquement « alignables ». Car si comme nous l'avions vu, nos trois interprétations de relire (ici assimilées à des points)...

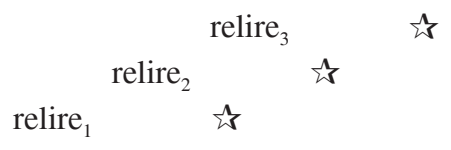

peuvent être décrites comme satisfaisant une même contrainte (ici assimilée à une droite) :

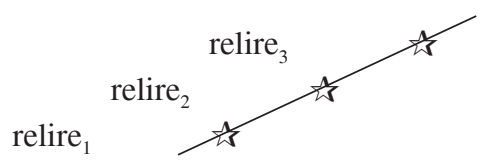

on observe donc que cette contrainte est satisfaite aussi par tous nos listèmes en re-, et par exemple par rechigner, rétorsion, répliquer, etc. et qu'il est de facto impossible de ne rendre compte que d'une partie des mots où $r e$ - est employé. Situation identique à celle qui prévaut quand après avoir trouvé une équation (ou une contrainte) satisfaite par $\mathrm{n}$ points (ou n observables), on découvre que la même équation (contrainte) est satisfaite par $\mathrm{n}$ autres objets qui ne faisaient pas partie de l'observable initial :

53 L'expression en contexte scolaire de «il retient bien » oppose ainsi la mémorisation p2 (qui fait que l'on se souvient de quelque chose) à la tendance naturelle à l'oubli p1. L'expression « retenir son souffle » oppose elle la tendance naturelle p1 à respirer et le fait p2 d'arrêter de le faire avant d'en faire un emploi métaphorique. « Retenir une somme » d'un salaire revient à « retrancher » cette somme du salaire, et donc à le « réduire », 


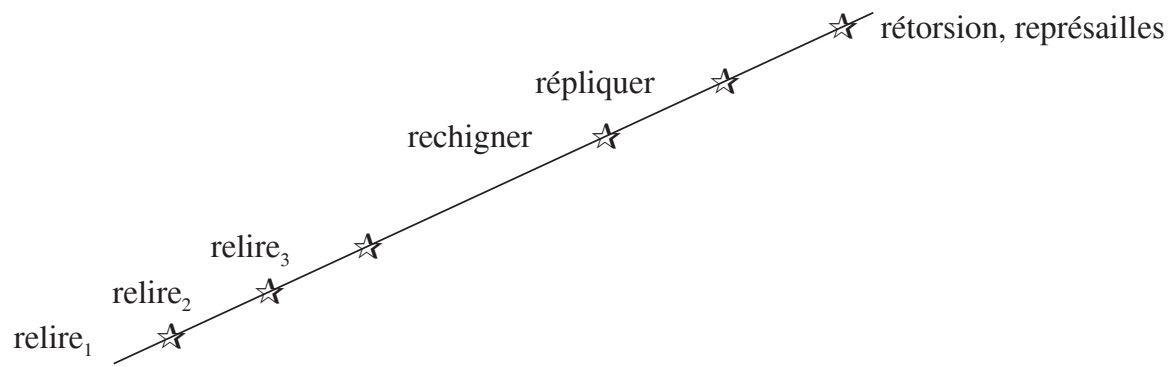

D'où il ressort par ailleurs que l'impossibilité grammaticale de rendre compte ou de prédire des mots comme rechigner ou se rebiffer ou réticent s'oppose en tout point avec le fait que ceux-ci sont parfaitement interprétables et ont des propriétés sémantiques prédictibles. Ce qui revient à dire que ces mots sont en fait sémantiquement bien formés et donc définis avant tout par leur interprétabilité, le linguiste n'ayant de ce fait pas à trier entre les «bons » mots contenant des lexèmes et les «mauvais » mots contenant autre chose (rotation, collision, rechigner, etc.) et devant reconnaître que tous les mots sont des objets sémantiques avant d'être des objets grammaticaux et qu'ils le demeurent même quand leurs composants ne sont pas des composants lexicaux. Et ce d'autant plus qu'il apparaît alors qu'ils sont aussi réguliers en français qu'un mot comme re-lire, et qu'il ne faut pas confondre le fait d'être réguliers et le fait que les « règles » en question soient à base de mots/lexèmes, ce qui est une tout autre question. Le fait qu'un verbe incluant $e n$ - en position dite suffixale n'ait pas de mot comme base (i.e. s'enticher vs s'enamourer) et que pour d'autres (s'emballer) le sens lexical du mot que l'on pourrait identifier comme la base ne soit pas sémantiquement pertinent, se retrouvant pour tous les suffixes du français et à des centaines d'occurrences à chaque fois, rien ne dit que ces trois patterns ne sont pas aussi réguliers les uns que les autres. Ceci à l'opposé presque exacte de l'hypothèse - dont la formulation la plus explicite est le précepte « one affix one rule $»^{54}$ - selon laquelle les morphèmes sélectionnent le type d'objets linguistiques (et notamment syntaxiques) avec lequel ils se

54 Trop radicale pour avoir jamais été pleinement acceptée sous cette forme, cette thèse présente néanmoins le mérite de rendre explicite ce qu'implique toute caractérisation par la grammaire d'une unité sémantique quelconque et qui continue à être réaffirmé à chaque fois que les considérations de stabilité grammaticale du morphème l'emportent sur les considérations sémantiques. Il faut noter que toute morphologie qui dans la constitution de son corpus d'observables définit un morphème par les unités avec lesquelles il se combine pratique de fait une version méthodologique de cette thèse : choisir de (ne) travailler (que) sur un préverbe ou sur des déverbaux revient en effet exactement à la même chose, et pèse lourdement sur la description sémantique. 
combinent. Puisqu'il s'avère donc que re- en tant que signe linguistique est combinable avec toutes sortes de constituants jouant eux-mêmes toutes sortes de rôle sémantique (y compris aucun rôle ou presque) et ce au terme d'une forme de mise en relation qui est purement sémantique.

\subsection{L'interprétabilité (et la polysémie) comme phénomènes exolexicaux et hololexicaux}

Ce qu'illustrent avant tout tous ces exemples, et la démonstration pourrait en français être élargie à des milliers d'emplois du morphème, c'est que les mots ne sont pas d'un point de vue sémantique et interprétatif des objets présentant une quelconque autonomie, et que de ce fait comprendre l'emploi d'un morphème ne se résume pour ainsi dire jamais à la seule mise en relation des différents composants du mot, les liens sémantiques à construire pour assurer son interprétation dépassant de très loin les limites du mot lui-même, et même du cotexte phrastique ou même discursif.

Ce qui fait de l'interprétabilité d'un mot une réalité qui dépasse nonseulement le mot mais même l'ensemble de l'explicite linguistique ce qui en fait un phénomène exolexical et pour une part exolinguistique ${ }^{55}$. Ce qui fait que les liens sémantiques quand ils restent endolinguistiques ${ }^{56}$ ressemblant bien plus à des lianes sémantiques qu'à quoi que ce soit qui suivrait les arbres grammaticaux, et il n'y a donc pour employer un langage montagovien aucune isomorphie entre construction grammaticale du mot et construction du sens, les listèmes n'étant pour l'essentiel que le retour de ce refoulé. Et ce qui fait enfin que la polysémie ne peut être étudiée véritablement qu'au niveau de l'ensemble des emplois du morphème et non au niveau des emplois d'un mot particulier, dès lors que ce qui apparaît inexplicable au niveau du mot isolé (e.g. le redire de "ne rien trouver à redire ») et qui est vite considéré comme de la « dérive sémantique », s'avère parfaitement explicable quand on rapproche l'interprétation concernée de l'ensemble des interprétations du morphème (tous mots confondus) avec lesquelles elle partage un même profilage et donc une même strate interprétative. D'où il suit que la polysémie est un phénomène hololexical qui ne peut s'étudier qu'au niveau d'un ensemble des mots et de leurs emplois, réalité que l'analyse et le découpage morphologique doivent intégrer, minable n'étant par exemple analysable qu'en relation (non dérivationnelle) avec minus (et avec le nom moins que rien).

${ }^{55} \mathrm{Du}$ point de vue de l'interprétation obtenue, il n'y a ainsi aucune différence entre l'interprétation par un français de camionneur et par un anglais de truck-driver, la seule différence étant que première construction est plus exolinguistique que la seconde (i.e. « exocentrique » dans la terminologie employée pour les composés).

56 Lien endolinguistique renvoie ici à tout lien entre un signe (morphémique ou lexical) et un élément ou ensemble d'éléments du cotexte, où que puissent se trouver ceux-ci. 
Le listage de la quasi-totalité du lexique n'est donc au total que la conséquence du fait que le modèle générativiste (comme toutes les approches combinatoires du mot) - et parfois une partie des tenants des morphologies constructionnelles - a tenu pour acquis (sans même en faire des axiomes ou hypothèses explicite) que tout élément dans un mot devait forcément ${ }^{57}$ avoir un sens (hypothèse de signifiance généralisée), que le sens d'un mot devait être obtenu à partir du sens des éléments qui le composent et de la seule association constructionnelle de ceux-ci entre eux (hypothèse d'endolexicalité) et enfin que le sens d'un mot (comme sa décomposition) pouvait être expliqué(e) sans étude distributionnelle complète de l'ensemble des éléments qui le composent, et était censé être obtenu par combinaison d'éléments locaux et non comme activation locale de patterns repérables ${ }^{58}$ seulement sur l'ensemble du lexique et de sa polysémie (hypothèse de micro-lexicalité). Loin d'être des « hors-la-loi », et de ne respecter aucune loi, il s'avère que ces pseudo-lois ne sont en réalité que des illusions théoriques et par conséquence qu'il faut bien reconnaître que ces listèmes supposés sont bien en réalité la norme linguistique en tant que celleci est régie in fine ${ }^{59}$ par les seules contraintes d'interprétabilité. Ce qui fait qu'il est indispensable de faire de leur étude et de leur théorisation l'objet de base de toute morphologie si celle-ci veut prétendre avoir quoi que ce soit à dire

${ }^{57}$ Ce qui semble être une conséquence non-maitrisée de la définition du morphème comme plus petite unité significative. Les linguistes semblent ainsi avoir beaucoup de mal à envisager que certaines parties d'un mot puissent ne pas être du tout signifiantes, et surtout que cela puisse ne poser aucun problème en ce qui concerne le mot en question. Admettre que le chign de rechigner puisse le cas échéant ne pas être signifiant, sans que cela bloque en quoi que ce soit l'interprétation du morphème re- apparaît impensable à beaucoup, alors qu'en parallèle beaucoup d'entre eux admettent l'existence de « morphèmes » dits cranberry qui illustrent pourtant le même phénomène. Sans pouvoir l'expliquer en détail, ces éléments mériteraient d'être appelés morphonèmes car si ce ne sont ni des signes lexicaux ni des signes morphémiques ils sont bien supports sémantiquement d'une valeur distinctive.

58 Si l'on considère les verbes français préfixés par en-, on observe ainsi qu'aussi imprédictibles que puissent paraître des mots comme emberlificoté, empêtré ou encastré ces mots relèvent en réalité de patterns de formation de mots extrêmement récurrents et ont une sémantique prédictible à partir des contraintes sémantiques imposées par le morphème en, à savoir notamment l'indication d'une perte de liberté de mouvement de quelque chose, que l'on retrouvera aussi bien dans des non-listèmes comme ensablé emprisonné ou encerclé, que dans nos trois listèmes ou encore dans entraîné ou s'enticher de, etc.

59 Le fait qu'une contrainte d'interprétabilité en vienne à générer des routines interprétatives qui à leur tout peuvent se grammaticaliser, formant ainsi des ilots de stabilité sémantique puis morphologiques, ne change rien au fait que c'est bien l'interprétation en tant qu'elle est régie par des contraintes purement sémantiques qui finira alors par se grammaticaliser. Au niveau du morphème tel que nous l'avons défini, aucune contrainte grammaticale ne préexiste aux emplois et en ce sens, ces contraintes sont toujours défaisables. Ce qui n'est pas le cas de la contrainte morphémique. 
sur le sens. Et qu'il est aussi important de comprendre que si la grammaire a échoué à en rendre compte, c'est sans doute parce que leur acceptabilité relève de contraintes avant tout sémantiques.

\subsubsection{Exolexicalité de l'interprétabilité et inexistence des sens construits}

S'agissant de comprendre l'exolexicalité de l'interprétabilité des signes et notamment des morphèmes (comme porteurs de contraintes sémantiques à satisfaire), elle tient donc d'abord à ce que l'interprétation (et le sens du mot quand celle-ci se lexicalise) n'est clairement pas réductible à la seule mise en relation des éléments qui le composent, qui ne sont d'ailleurs pas tous des éléments sémantiques ${ }^{60}$. Ce qui signifie à l'inverse que pour interpréter un seul de ces éléments l'interprétant devra chercher aussi loin que nécessaire ce que les instructions/indications sémantiques lui imposent de chercher et de trouver ${ }^{61}$ et qu'il n'existe donc en matière d'interprétabilité d'un élément sémantique d'un mot aucune contrainte de proximité qui limiterait l'interprétation à la limite du mot ou même de la phrase énoncée. Réalité qui fait que tout modèle postulant de façon tacite ou explicite, l'existence d'une sorte d'atelier de construction des mots et de leurs sens, où ceux-ci seraient dotés d'un sens par mise en relation directe des seuls composants du mot, se heurtera au constat inévitable d'une forte instabilité de la relation sémantique visée, et ce même si par ailleurs l'interprétation globale associée au mot s'avère elle très largement prédictible. Le sémanticien et le morphologue ne doivent donc absolument jamais conditionner la reconnaissance d'un composant du mot et la description de l'interprétation qui lui est associée à l'identification de tous les composants du mot ou à celle d'une relation stable entre ceux-ci : comprendre ressuscité ou se rétracter ne passe pas par une décomposition de ces mots en éléments signifiants associables entre eux selon un plan invariable mais passe directement, en ce qui concerne le morphème $r e$ - par l'identification d'un processus $\mathrm{p} 1$ ( " passage de vie à trépas », « avouer ») suivi d'un processus inverse («passage de trépas à vie », « revenir sur ses aveux »). Moyennant quoi l'interprétabilité de ces deux mots n'est aucunement affectée par le caractère au mieux opaque des autres composants et de leur rôle sémantique, que le linguiste devra in

${ }^{60}$ Ce qui fait que l'interprétabilité n'impose même pas une mise en relation sémantique obligatoire des éléments en question, et de ce fait rend possible que certains de ces éléments soient non-signifiants. Situation semblable à celle de l'ADN pour faire un parallèle, dont des séquences entières sont non-codantes.

61 Dans l'interprétation d'un énoncé aussi simple que «ils sont célèbres pour avoir repousséles vikings » par le lecteur de cet article, celle-ci a ainsi toutes les choses de passer par l'identification des vikings comme agents du processus p1 qui doit être trouvé, et ce faisant, en l'espèce sur la base d'un savoir partagé non co-textuel sur les Vikings, faire l'hypothèse que p1 est une attaque menée par ceux-ci. Ce que rien de linguistique n'impose. 
fine expliquer mais qu'il n'a pas à expliquer ou justifier a priori pour pouvoir reconnaître la présence du morphème. Ce qui lui interdit, à l'exact opposé de la thèse du listage, d'écarter de ce dont il doit rendre compte, les mots (et sens) qu'il ne peut pas initialement complètement expliquer ${ }^{62}$. Mais ce qui fait que si morphologues et sémanticiens n'ont donc pas à résoudre du premier coup l'ensemble de ce dont ils auront à rendre compte ensuite, ils ont en revanche à rendre compte de l'ensemble des interprétations des mots concernés, aussi diverses que celles-ci puissent être et ne peuvent en aucun cas sélectionner telle ou telle interprétation pour en faire le sens de base du mot concerné en listant ensuite tous les autres sens. La réalité étant que les mots, au sens purement syntaxique du terme, n'ont pas de sens en tant que tels mais seulement dans leurs emplois, quand ils se transforment en usages et qu'il y a lexicalisation mémorisation et conventionnalisation des interprétations associées à ces usages.

Sans rentrer ici dans le détail considérable de tous les aspects sémantiques de cette question, il faut souligner que si comme nous l'avons vu il s'avère impossible de rendre compte de la polysémie de mots apparemment «simples et transparents » comme relire, redire, redonner sans rendre compte ipso facto de notre liste de listèmes, les choses vont en fait plus loin que cela, dès lors que la polysémie de nos ex-listèmes, y compris ce qui concerne les « dérivés » de ceux-ci, s'avère elle aussi explicable en termes de satisfaction d'une unique contrainte morphémique. Ce qui revient à dire d'une part que le lien sémantique démontrable existant entre tous les sens ${ }^{63}$ de redire est identique à celui existant entre tous les sens de retenir (en l'espèce donnant lieu ou non à listage : « il a été retenu par le collet» vs «il s'est retenu de l'insulter» ou «j'ai eu du mal à retenir mes leçons d'histoire ».), ou encore les très nombreux sens de rendre ou de restitution, etc. Et ce qui revient à dire d'autre part qu'il y aura des liens interprétatifs directs entre tel emploi/sens d'un mot donné et un ensemble d'emplois distribués sur un ensemble de mots partageant tous un interprétation-type, comme variante spécifique de l'indication morphémique. Ainsi l'explication de la diversité des emplois de « revanche », y compris

62 Il est particulièrement paradoxal que le mot répétition, illustration s'il en est du fait que le sens d'un mot puisse être parfaitement prédictible alors que le chemin qui mène à cette interprétation reste opaque, puisse être utilisé comme outil de description métalinguistique de la signification de re, tout en étant listé par ailleurs. Et ce alors même que ce mot contenant le morphème re-, il ne devrait par définition pas jouer ce rôle pour la même raison qui interdit au linguiste d'utiliser la notion de surface dans toute description de sur, dès lors que sur étant dans surface mais face n'y étant pas, surface ne peut pas être dans la signification morphémique de sur. La signification d'un signe ne peut pas être associée à l'interprétation d'un mot complexe dans lequel il est inséré.

${ }^{63}$ En l'espèce, aussi bien des sens présents (trouver toujours à redire, etc.) que des sens passés tels que « dire à son tour», « révéler ce qu'on a appris de quelqu'un en confidence », etc. 
l'emploi discursif «en revanche » passera-t-elle inévitablement par l'antiorientation morphémique et l'idée que l'inverse de quelque chose, victoire ou toute autre chose, devient vrai. Et il sera ainsi possible d'isoler dans les emplois de redire ceux qui relèvent d'une interprétation-type de récurrence au sens large, ou d'une interprétation-type impliquant une forme d'opposition ${ }^{64}$ à quelque chose ("personne n'y a rien trouvé à redire ») ou encore d'autres interprétations-types (e.g. un emploi disparu paraphrasable par «dire à son tour »). Alors que la polysémie de rendre quant à elle situera certains emplois du côté de restituer (ou même de redonner) et d'autres du côté de reddition ${ }^{65}$, etc. Et ce sans que la distinction entre mots listés et mots listés ou entre sens listés et sens non listés ait la moindre importance interprétative. Moyennant quoi, le linguiste se faisant sémanticien pourra par exemple identifier d'abord comme domaine d'emploi du morphème $r e$ - un type de situation où deux forces opposées s'exercent simultanément, l'une l'emportant néanmoins souvent sur l'autre (qu'elle peut alors masquer ${ }^{66}$ ) et identifier ensuite l'ensemble des observables pour lesquels tout (restriction renâcler, regimber, rétif) ou partie (se retenir de, refreiner, rétention) des emplois d'un mot ressort de ce type de situation. Le tout sans que ces regroupements par profils sémantiques puissent constituer un stade ultime de la description polysémique, dès lors par exemple que dans les emplois-types de récurrence, il faudra ensuite distinguer la répétition avec successivité temporelle (testable par exemple par la glose « une nouvelle fois ») et la répétition sans successivité temporelle que l'on observe dans l'emploi linguistique de « reduplication » (qui ne s'interprète pas comme «dupliquer une nouvelle fois », mais plutôt comme "présent en deux exemplaires ») ». Et sachant par ailleurs que le mot « répétition » lui-même s'avérera employable pour parler aussi bien de "quelque chose qui succède à quelque chose » que de "quelque chose qui précède quelque chose » (la répétition du théâtre ou d'un spectacle) ${ }^{67}$.

Cette plasticité de l'interprétation est à l'évidence ce qui a le plus dérouté les linguistes quand ils y ont été confrontés, et la raison pour laquelle autant d'entre eux ont choisi de minimiser ou de simplifier les questions sémantiques. Elle ne doit cependant pas faire illusion : aussi subtiles que soient les nuances

64 Au coté, en variante faible, de reprocher et remontrance.

65 Pour certains des emplois du mot, tandis que d'autres emplois tels que « reddition d'une somme d'argent » se situeront eux du côté de rembourser, restituer et... d'un des sens de rendre. main ».

${ }_{66}$ Profil que l'on peut illustrer par l'image de quelqu'un qui « roule avec le frein à

${ }^{67}$ On peut ainsi dire «nous n'avons pu répéter qu'une seule fois » pour parler de la préparation d'une pièce, le mot « répétition » désignant bien dans ce cas la première des deux phases et non la seconde. 
qu'il faut faire pour rendre compte de façon un peu sérieuse du sens lexicalisé des constructions morphologiques, il reste que l'arbre des interprétations d'un morphème que l'on peut décrire dès lors que l'on considère l'ensemble des mots et de leurs emplois où celui-ci est utilisé, apparaît réductible à un nombre fini de profils ${ }^{68}$, qui certes sont aussi arborescents mais qui réunissent néanmoins chacun un très grand nombre d'emplois. Il faut enfin signaler que toute représentation de la polysémie lexicale reposant obligatoirement sur des tests sémantiques, les relations entre signification morphémique (testable), profils (testables), sous-profils (testables) et sens (interprétations lexicalisées) sont d'une part des relations d'héritage (au sens informatique et mathématique du terme) puisqu'un profil par exemple héritera obligatoirement des propriétés morphémiques, qui seront donc des propriétés partagées, et d'autre part des relations de différentiation, puisqu'il y aura dans chaque profil des propriétés non morphémiques. Moyennant quoi, il devient possible de regrouper tous les emplois qui partagent un même profil ou sous-profil mais aussi de décrire d'un point de vue sémantique à la fois les propriétés partagées par deux emplois et les propriétés non-partagées. Un peu comme si quelqu'un ayant à établir les relations entre deux feuilles quelconque d'un arbre (deux emplois) était conduit à devoir chercher à quel niveau (tronc, branches maitresses, branches, branchettes) les deux feuilles en question sont en relation, et l'ensemble des propriétés partagées comme des propriétés différentielles.

Ce qui fait que l'on aura non pas une signification et un ensemble de sens autonomes les uns des autres, comme dans le schéma 1 :

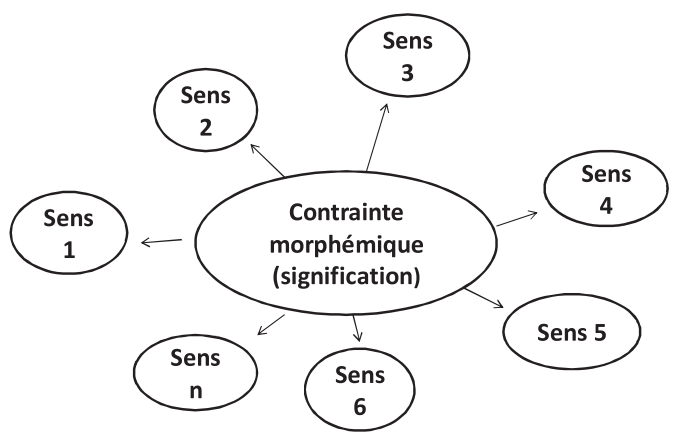

${ }^{68}$ Qui sont l'équivalent lexical des branches maitresses d'un arbre, et comme elles peuvent se ramifier à leur tour en sous-profils, etc. 
mais un ensemble de profils et sous profils distincts ${ }^{69}$ comme dans le schéma $2^{70}$ :

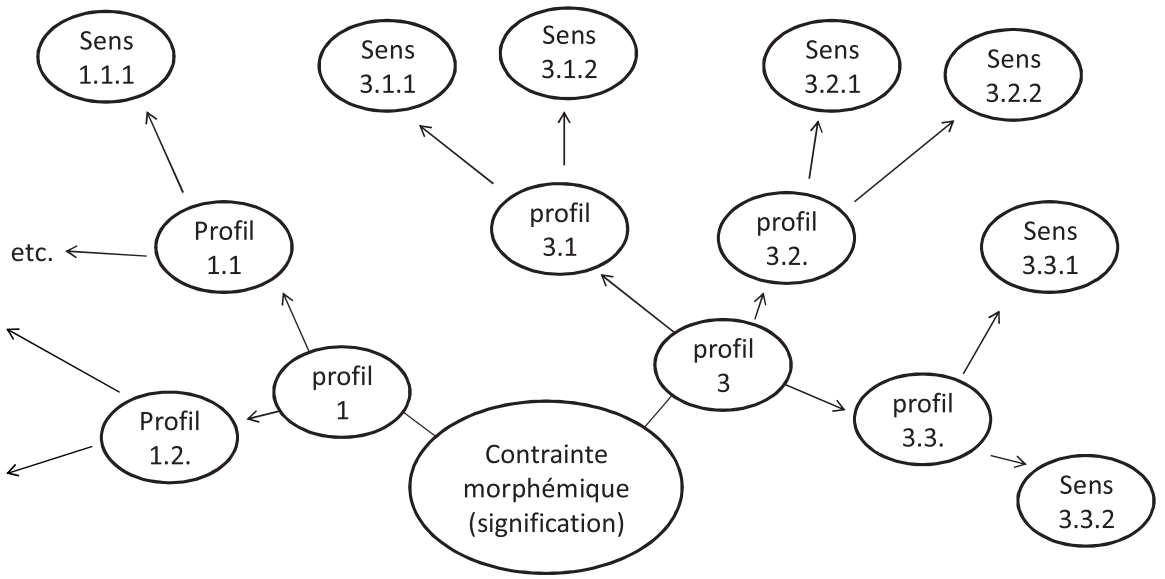

Faire de la sémantique en morphologie suppose donc d'étudier l'ensemble des interprétations d'un seul coup et de s'interdire de transformer une quelconque d'entre elles, et particulièrement les plus intuitives, en sens premier. Les données, autrement dit les faits linguistiques, n'étant à cet égard nullement équivoques, et montrant, à l'instar de séries comme regret, remord, se repentir, etc. qu'une interprétation stable n'est jamais conditionnée par le statut grammatical et même sémantique de la base. Ceci, non seulement parce que la base du mot n'apporte pas toujours la même information (Nemo, 2010, à propos de déterrer et désherber), mais parce qu'il n'apporte parfois aucune information, sans que cela bloque en quoi que ce soit l'interprétabilité de l'ensemble ${ }^{71}$. Il devient alors possible pour un morphème comme re- de se combiner avec un nom, comme dans recycler, et ce sur une base strictement sémantique, à savoir le fait qu'un cycle est un processus dans lequel se succèdent deux étapes anti-orientées (nos fameux p1 et p2) où le point d'arrivée de la seconde avait été initialement le point

69 Pour une illustration détaillée de la notion de profil lexical, voir Petit \& Nemo 2010. A noter que la notion de profilage s'applique ici bien à la signification codée par un morphème (non intuitif), dont elle procède, et ne concerne pas les «meaning profiles » des mots et de leur référent comme en sémantique cognitive (Givon, Evans).

70 Schéma dans lequel chaque flèche décrit une relation d'héritage sémantique et chaque niveau une strate sémantique, avec comme résultat un sens final pluristratique

71 À la manière où dès lors qu'est fournie (par l'affixe) l'équation à résoudre (la contrainte à satifaire), le fait que la base fournisse ou non telle ou telle des inconnues n'empêche jamais de trouver ce que l'on cherche et rend secondaire la question de savoir à quel endroit elle est trouvée ou encore si elle se trouve dans la base du mot. 
de départ de la première. Moyennant quoi ce sera ce seul lien d'unification entre cycle et (p1p2) qui rendra possible le listème et son interprétation, et expliquera la non-nécessité de l'existence d'une règle grammaticale de type WFR.

On peut alors comprendre simultanément :

- le fait qu'un même morphème puisse être associé à un ensemble de constructions et la relation exacte entre ces constructions, question fondamentale pour toute grammaire de construction ;

- le fait que la signification du morphème ne doit jamais être réduite à la description de l'interprétation de telle ou telle des constructions concernées ;

- le fait que tous les lexèmes comportant le même morphème forment un complexe sémantique ${ }^{72}$ qui doit être étudié en tant que tel ;

- le fait que l'existence de constructions n'implique en rien que le sens soit construit et qu'il doive exister un sens construit, mais seulement que les constructions peuvent contribuer à l'interprétation et doivent être caractérisées comme faisant partie des inputs du processus interprétatif (des contraintes à satisfaire) et non en termes de résultat de celui-ci, qui n'est lui véritablement prédictible qu'à partir des instructions morphémiques $^{73}$.

Important aussi est le fait que les constructions morphologiques fonctionnent sémantiquement comme un jeu de chaises musicales, avec un nombre d'arguments sémantiques plus grands que le nombre de positions structurales disponibles ${ }^{74}$, avec la possibilité que différents arguments occupent alternativement la même position structurale sans que l'interprétation finale

$72 \mathrm{La}$ coexistence d'unités porteuses de signification et d'unités porteuses d'interprétation lexicalisée (sens) implique l'existence de tels complexes qui associent chaque unité porteuse de signification à l'ensemble de ses emplois lexicalisées. Le lexique n'est donc pas une liste, et les ex-listèmes ne sont donc pas des unités isolées à apprendre une par une mais bien, l'hypothèse est psycholinguistiquement testable, des unités associées à des réseaux morphosémantiques.

73 Celles-ci n'étant jamais « mariées » à une construction particulière.

74 Comme on peut l'observer dans toute comparaison du français et de l'anglais en ce qui concerne les composés synthétiques (truckdriver vs camionneur), le mot français ne contenant pas conduire qui est pourtant aussi nécessaire à son interprétation que dans le mot anglais. Ce qui illustre une nouvelle fois le caractère exolexical de l'interprétation et le fait que décrire camionneur comme un dérivé ne veut sémantiquement rien dire. Tout comme ne voudrait rien dire le fait de devoir postuler (Aronoff, 2004, 3) deux morphèmes -eur en français selon qu'il y a combinaison avec un verbe ou un nom, ce nom n'étant sémantiquement que le COD d'un verbe implicité (à trouver) qui dans chanteur est trouvé dans la base (cf. Nemo, 2010). 
change en quoi que ce soit ${ }^{75}$. Ou encore qu' aucun argument sémantique n'occupe une position structurale disponible, là aussi sans bloquer l'interprétation (et l'interprétabilité $)^{76}$. Et avec la possibilité enfin d'insérer dans une position structurale aussi bien des lexèmes (re-lire), des morphèmes (re-st-er $)^{77}$ que des unités asémantiques.

\section{CONCLUSION}

Parler de l'interface entre deux disciplines ou deux domaines ne consiste pas à confronter les a priori de chacune d'entre elles mais à définir les questions qu'elles peuvent et doivent traiter ensemble. Dire que la morphologie plus que tout autre domaine a été confrontée à la difficulté de comprendre la nature des mécanismes qui gouvernent l'interprétation et la mise en relation sémantique des constituants linguistiques est une litote, tant la tache était complexe et tant les outils utilisés initialement pour le faire étaient en réalité inadaptés. Le fait qu'un même morphème puisse être associé à un ensemble de constructions, dont certaines (pourtant très productives) sont aussi inaccessibles à l'intuition lexicale ou grammaticale qu'une maison en torchis peut l'être d'une maison en brique, le fait aussi que sa signification ne soit jamais réductible à l'interprétation qu'il peut avoir dans telle ou telle des emplois et constructions concernées - et en particulier dans ses emplois dits libres qui sémantiquement n'ont rien de premier - telles sont avec la question des chaises musicales quelques unes des causes qui conduisent au listage de la plus grande partie du lexique.

Le fait qu'une compréhension de plus en plus fiable de la nature des mécanismes sémantiques permet aujourd'hui de rendre compte de réalités linguistiques qui jusqu'ici semblaient devoir être caractérisées avant tout par leur « irrationalité » apparente, a ouvert à l'évidence un nouveau chapitre de la sémantique mais aussi de la morphologie. Ce qui est d'autant plus vrai que comme nous l'avons vu rien ne permet de penser que sélectionner les données morphologiques sur des bases structurales ou intuitives, comme on a pu être

75 Comme on vient de le voir avec le contraste entre déterrer et désherber pour lesquels on a un profil d'interprétation unique « $\mathrm{X}$ est retiré de $\mathrm{Y}$ » et deux routines interprétatives distinctes « dé-Y-er X » (retirer le trésor de la terre) et « Dé-X-er Y » (retirer l'herbe du jardin). Il en est de même avec prisonnier qui inverse dans le profil « $\mathrm{X}$ est dans $\mathrm{Y} »$, la relation la plus fréquente « $\mathrm{X}$ est dans $\mathrm{Y}$ » (i.e. « Les cloches sont dans le clocher») par la relation «Y est dans $\mathrm{X} »$ (i.e. « les prisonniers sont dans la prison»). Cf Nemo (2002b, 2010).

${ }^{76}$ Comme on le voit avec rétif pour lesquels ni p1 ni p2 ne sont inférables à partir de tif et ce sans que cela empêche en quoi que ce soit de trouver tel ou tel argument sémantique dans le cotexte (rétif à l'idée de s'engager », avec p1 = s'engager) ou ailleurs.

${ }^{77}$ Rester reste en effet associé à une routine interprétative re-P2, le morphème st (que l'on retrouve dans l'anglais stay et dans de très nombreux mots appartenant aux langues romanes, dont le français, cf. le latin stare) décrivant bien le p2 recherché. 
tenté de le faire pendant longtemps, puisse permettre d'obtenir de meilleurs résultats, y compris en ce qui concerne le traitement des données sélectionnées elles-mêmes.

C'est pourquoi les listèmes, après avoir été les pestiférés de la morphologie du fait de leur anormalité apparente, peuvent devenir au contraire un terrain extraordinaire pour saisir dans sa complexité et la question de la diversité des emplois des signes et celle au moins aussi complexe de la façon dont ceux-ci sont associables car co-interprétables.

Sémanticiens et morphologues disposent de ce point de vue d'un immense avantage, qui est que l'interprétation est - à condition d'en avoir une vision ouverte - objectivable par quiconque y consacre le temps nécessaire et accepte de renoncer à l'intuition. L'interface entre sémantique et morphologie s'annonce de ce point vue riche en découvertes à venir et doit devenir pour la linguistique une nouvelle frontière. 


\section{BIBLIOGRAPHIE}

Aronoff, Mark (1976). Word Formation in Generative Grammar. Cambridge (Ma): the MIT Press.

Aronoff, Mark.\& Anshen Franck (1998). « Morphology and the Lexicon ». In Spencer Andrew \& Zwicky Arnold (eds.), The Handbook of Morphology. Oxford: Blackwell, pp. 248-271.

Bar-Hillel, Yehoshua (1971) « Out of the Pragmatic Wastebasket », Linguistic Inquiry, II, $n^{\circ} 3$, pp. 401-406.

Benveniste, Emile (1954). " Problèmes sémantiques de la reconstruction », Word, X, 2-3, repris dans Problèmes de linguistique générale, 1, 1966. Paris, Gallimard, pp. 289-307.

Bybee, Joan (1988). « Morphology as Lexical Organization ». In Hammond, Michael \& Noonan Michael (eds.) Theoretical Morphology: Approaches in Modern Linguistics. San Diego: Academic Press. pp. 119-141.

Cadiot Pierre \& Viestti Yves-Marie (2001), « Pour une théorie des formes sémantiques ». Paris ; PUF Carstairs-MacCarthy, Andrews (2005). «Basic Terminology». In the Handbook of Word Formation. (Štekauer, Pavol \& Lieber, Rochelle ; Eds) Dordrecht : Springer. 5-24.

Corbin, Danielle (1987). Morphologie dérivationnelle et structuration du lexique. Tübingen; Max Niemeyer. Verlag..

Corbin, Danielle \& Corbin Pierre (1991) «Un traitement unifié du suffixe -ier(e)». Lexique, 10. 61-145.

Di Sciullo, Anna Maria \& Williams Edwin (1987). On the Definition of Word. Cambridge: the MIT press

Dixon, Robert \& Aikhenvald, Alexandra (2002). Word: A Cross-linguistic Typology. Cambridge: Cambridge University Press.

Ducrot, Oswald (1987). «L'interprétation comme point de départ imaginaire de la sémantique ». In Dire et ne pas dire ([1972], 1991). Paris; Hermann.

Fradin, Bernard (1999), Syntaxe et morphologie. Histoire Epistémologie et Langage. XXI, 2, pp. 7-44.

Goldberg, Adele (1995). Constructions. Chicago: University of Chicago Press.

Jalenques, Pierre (2000). Contribution à l'étude du préfixe RE en français contemporain: pour une analyse compositionnelle du verbe regarder. Thèse de doctorat, Paris 7.

Hengeveld, Kees (1992). Non-verbal predication. Theory, Typology, Diachrony. Berlin/ New York : Mouton de Gruyter.

Levin, Beth \& Rappaport Hovav M (1998). « Morphology and Lexical Semantics ». In A. Spencer \& A. Zwicky (eds.) The Handbook of Morphology. Oxford: Blackwell, pp. 248-271. 
Levinson, Stephen (2000). Presumptive Meanings. The Theory of Generalized Conversational Implicature. Cambridge: the MIT Press.

Lieber, Rochelle (1992). Deconstructing Morphology: Word Formation in Syntactic Theory. Chicago: University of Chicago Press.

Nemo, François (2001a). « Pour une approche indexicale (et non procédurale) des instructions sémantiques ». Revue de Sémantique et Pragmatique. Numéro 9-10, pp. 195-218.

Nemo, François (2001b). Contributions, énoncés, constructions, morphèmes. Eléments pour une linguistique de la signification et de l'interprétation. Thèse d'Habilitation à diriger des recherches. Paris 8.

Nemo, François (2002a). "De la génération du sens. Remarques sur la sousdétermination ». Revue de Sémantique et Pragmatique,12, pp 7-15.

Nemo, François (2002b). « Morpheme Semantics and the Autonomy of Morphology. The Stable Semantics of (Apparently) Unstable Constructions » in Andronis, Mary, Christopher Ball, Heidi Elston and Sylvain Neuvel eds. CLS 37: The Panels. Papers from the 37th Meeting of the Chicago Linguistic Society. Vol. 2. Chicago: Chicago Linguistic Society.

Nemo, François (2003) «Indexicalité, unification contextuelle et constitution extrinsèque du référent », Langages, juin, ${ }^{\circ} 150$, pp. 88-105.

Nemo, François (2004) «Constructions et morphèmes : réflexions sur la stabilité en sémantique », Revue de Sémantique et Pragmatique. Numéro 15-16, pp 19-35.

Nemo, François (2005). « Morphemes and Lexemes versus Morphemes or Lexemes ». In on-line Proceedings of the 4th Mediteranean Morphology Meeting (MMM4) on Morphology and Linguistic Typology. (Catania, Sicile, 21-23 Septembre 2003). Booij, Guevara, Ralli, Sgroi \& Scalise (eds). Université de Bologne. ISSN 1826-7491

Nemo, François (2006a). «Contre la modularité ». Revue de Sémantique et Pragmatique, numéro $19-20$, pp. 27-50

Nemo, François (2006b). « Discourse words as morphemes and as constructions ». In Approaches to Discourse Particles (Studies in Pragmatics, Vol 1). Kerstin Fischer (Ed). Amsterdam : Elsevier Science. pp. 415-448.

Nemo, François (2007). "Reconsidering the Discourse Marking Hypothesis ». In Connectives As Discourse Landmarks (Pragmatics and Beyond New Series) Celle A \& Huart, R (Eds). Amsterdam-Philadelphia: John Benjamins Publishing. pp. 195-210.

Nemo, François (2010) « Routines interprétatives, constructions grammaticales et constructions discursives ». Estudos Linguísticos/Linguistic Studies, 5, Edições Colibri/CLUNL, Lisboa, pp.35-53. 
Plag, Ingo. (1998), « The Polysemy of -ize Derivatives. The Role of Semantics in Word Formation », in G. Booij \& J. van Marle (eds.) Yearbook of Morphology 1997. Dordrecht: Kluwer. pp. 219-242.

Pustejovsky, James. (1995) The Generative Lexicon. Cambridge (Ma): The MIT press. Temple, Martine (1996). Pour une sémantique des mots construits. Lille ! Presses du Septentrion.

Nemo, François (2007a). « Reconsidering the Discourse Marking Hypothesis ». In Connectives As Discourse Landmarks (Pragmatics and Beyond New Series) A. Celle \& R. Huart (Eds). Amsterdam/Philadelphia: John Benjamins Publishing. 195-210.

Nemo, François (2007b). « La sémantique peut-elle être catégorielle ? ». In La représentation du sens en linguistique 2. D. Bouchard, I. Evrard, et E. Vocaj (Eds). Bruxelles : Boeck-Duculot. 35-52.

Pelletier, Francis J (1994) «The Principle of Semantic Compositionality » Topoi 13: 11-24. . Repris dans S.Davis \& B. Gillon (eds.) 2004 Semantics: A Reader. Oxford University Press. 133-156.

Robert, Stéphane (2003) Perspectives synchroniques sur la grammaticalisation: polysémie, structure mie, transcatégorialité et échelles syntaxiques. Louvain : Peeters.

Sapir, Edward (1944). «Grading : A Study in Semantics ». In Philosophy of Science, 11: 93-116. [Réinprimé in: D.G. Mandelbaum (ed.), 1963. Selected Writings of. Edward Sapir in Language, Culture and Personality. Berkeley: University of California. Press. 122-149.]

Scalise, Sergio \& Guevara, Emiliano (2005). « The Lexicalist Approach to WordFormation and the Notion of the Lexicon ». In the Handbook of Word Formation. (Štekauer, Pavol \& Lieber, Rochelle ; Eds) Dordrecht : Springer. 147-187. 